\title{
Radiological Conditions at Naen, Yugui, Lomiulal, Kabelle and Mellu Islands in the Northern Half of Rongelap Atoll
}

\author{
William L Robison \\ Cynthia L. Conrado
}

\section{PECEVED \\ JUL 22 1996 \\ OSTI}

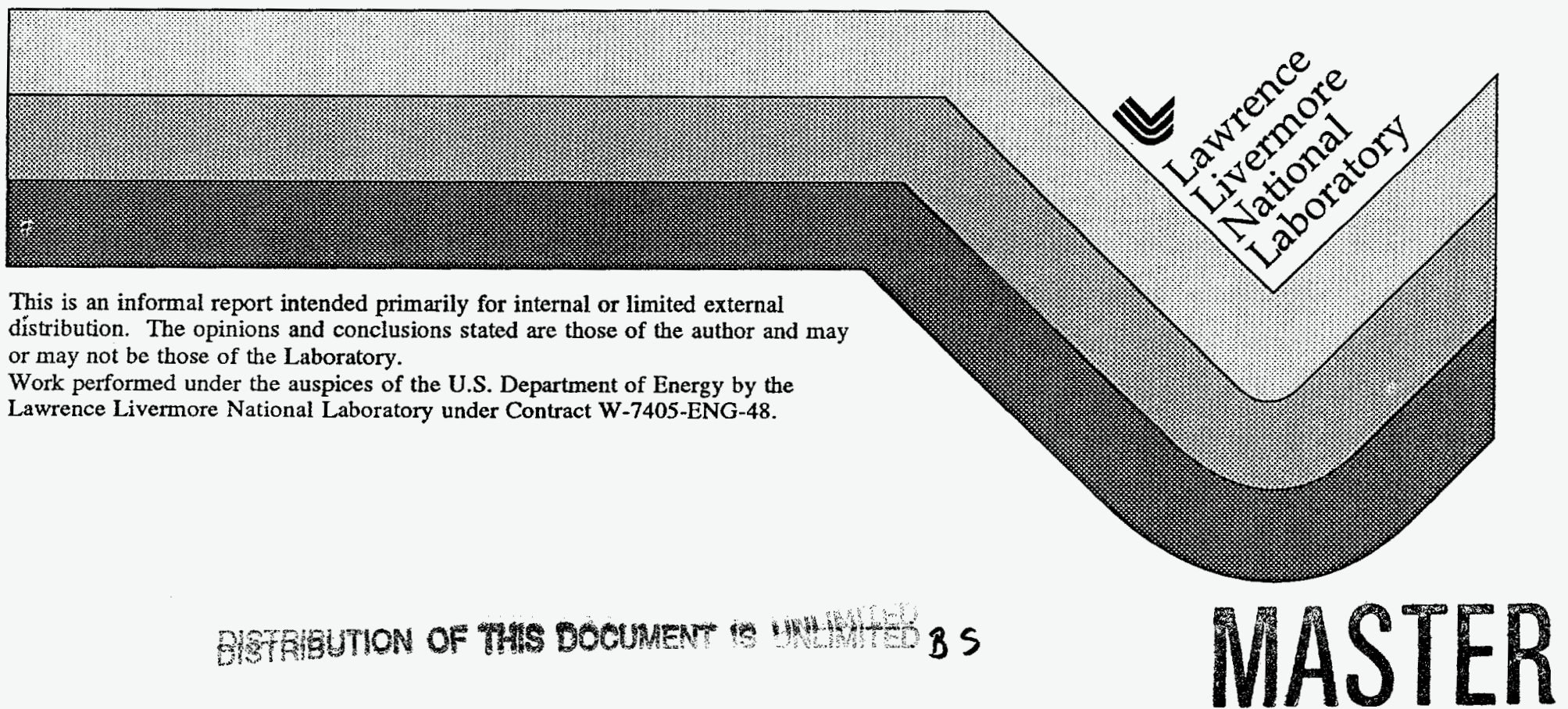




\section{DISCLAIMER}

This document was prepared as an account of work sponsored by an agency of the United States Government. Neither the United States Government nor the University of Califomia nor any of their employees, makes any warranty, express or implied, or assumes any legal liability or responsibility for the accuracy, completeness, or usefulness of any information, apparatus, product or process disclosed, or represents that its use would not infringe privately owned rights. Reference herein to any specific commercial product, process, or service by trade name, trademark, manufacturer, or otherwise, does not necessarily constitute or imply its endorsement, recommendation, or favoring by the United States Govemment or the University of California. The views and opinions of authors expressed herein do not necessarily state or reflect those of the United States Government or the University of California, and shall not be used for advertising or product endorsement purposes.

This report has been reproduced directly from the best available copy.

Available to DOE and DOE contractors from the Office of Scientific and Technical Information P.O. Box 62, Oak Ridge, TN 37831

Prices available from (615) 576-8401, FTS 626-8401

Available to the public from the

National Technical Information Service

U.S. Department of Commerce

5285 Port Royal Rd.

Springfield, VA 22161 


\section{DISCLAIMER}

Portions of this document may be illegible in electronic image products. Images are produced from the best available original document. 

Radiological Conditions at Naen, Yugui, Lomiulal, Kabell and Mellu Islands in the Northern Half of Rongelap Atoll

\author{
William L. Robison \\ Cynthia L. Conrado \\ Lawrence Livermore National Laboratory \\ Environmental Sciences Division
}

Report to DOE Environment, Safety, and Health for Dr. Harry Pettengill, March 1992

March, 1996 


\section{TABLE OF CONTENTS}

Introductory Comments.

.01

Table 1 The number of food crops collected at the northern islands at Rongelap Atoll and the mean and median ${ }^{137} \mathrm{Cs}$ concentrations.

Table 2 The number of indicator species collected at the northern islands at Rongelap Atoll and the mean and median ${ }^{137} \mathrm{Cs}$ concentrations........03

Table 3 The number of animal samples collected at the northern islands at Rongelap Atoll and the mean and median ${ }^{137} \mathrm{Cs}$ concentrations........04

Table 4 The number of soil samples collected at the northern islands at Rongelap Atoll. .05

Table 5 Average ${ }^{137} \mathrm{Cs},{ }^{90} \mathrm{Sr},{ }^{239}+240 \mathrm{Pu}$, and ${ }^{241} \mathrm{Am}$ soil concentrations in $\mathrm{pCi} / \mathrm{g}$ dry weight for soil profiles taken on Naen Island (F1)............................06

Table 6 Average ${ }^{137} \mathrm{Cs},{ }^{90} \mathrm{Sr},{ }^{239+240} \mathrm{Pu}$, and ${ }^{241} \mathrm{Am}$ soil concentrations in $\mathrm{pCi} / \mathrm{g}$ dry weight for soil profiles taken on Yugui Island (F5)..........................06

Table 7 Average ${ }^{137} \mathrm{Cs},{ }^{90} \mathrm{Sr},{ }^{239}+240 \mathrm{Pu}$, and ${ }^{241} \mathrm{Am}$ soil concentrations in $\mathrm{pCi} / \mathrm{g}$ dry weight for soil profiles taken on Lomiulal Island (F7) ....................07

Table 8 Average ${ }^{137} \mathrm{Cs},{ }^{90} \mathrm{Sr},{ }^{239+240} \mathrm{Pu}$, and ${ }^{241} \mathrm{Am}$ soil concentrations in pCig/ dry weight for soil profiles taken on Kabelle Island (F13)......................07

Table 9 Average ${ }^{137} \mathrm{Cs},{ }^{90} \mathrm{Sr},{ }^{239+240} \mathrm{Pu}$, and ${ }^{241} \mathrm{Am}$ soil concentrations in $\mathrm{pCi} / \mathrm{g}$ dry weight for soil profiles taken on Mellu Island (F23)........................08

Figure $1 \quad$ 137Cs in soil at Rongelap Atoll-northern islands............................09

Appendix ARadionuclide concentration summary in vegetation samples collected during the 1978 NMIRS and from 1986 through 1989 in the northern islands of Rongelap Atoll. A-10

Table A-1 The ${ }^{137} \mathrm{Cs}$ radionuclide concentration summary for vegetation (food crops) collected from the northern islands of Rongelap Atoll during the 1978 NMIRS together with our recent trips in 1986 through 1989.. A-11

Table A-2 The ${ }^{137} \mathrm{Cs}$ radionuclide concentration summary for vegetation (indicator species) collected from the northern islands of Rongelap Atoll during the 1978 NMIRS together with our recent trips in 1986 through 1989. 
Appendix B Radionuclide concentration summary in animal samples collected during the 1978 NMIRS and from 1986 through 1989 in the northern islands of Rongelap Atoll.

Table B-1 The ${ }^{137} \mathrm{Cs}$ radionuclide concentration summary for animals (food source) collected from the northern islands of Rongelap Atoll during the 1978 NMIRS together with our recent trips in 1986 through 1989.

Appendix C Radionuclide concentration summary of all soil profile samples collected during the 1978 NMIRS and from 1986 through 1989 in the northern islands of Rongelap Atoll

Table C-1 Cesium-137 radionuclide concentration summary for all soil profiles taken from the 1978 NMIRS together with our most recent trip in 1989 on Naen Island (F1).

Table C-2 Cesium-137 radionuclide concentration summary for all soil profiles taken from the 1978 NMIRS together with our most recent trip in 1989 on Yugui Island (F5).

Table C-3 Cesium-137 radionuclide concentration summary for all soil profiles taken from the 1978 NMIRS together with our most recent trip in 1989 on Lomiulal Island (F7). C-18

Table C-4 Cesium-137 radionuclide concentration summary for all soil profiles taken from the 1978 NMIRS together with our most recent trips in 1988 through 1989 on Kabelle Island (F13). C-19

Table C-5 Cesium-137 radionuclide concentration summary for all soil profiles taken from the 1978 NMIRS together with our most recent trip in 1989 on Mellu Island (F23). C-20

Table C-6 Strontium-90 radionuclide concentration summary for all soil profiles taken during the 1978 NMIRS on Naen Island (F1). C-21

Table C-7 Strontium-90 radionuclide concentration summary for all soil profiles taken during the 1978 NMIRS on Yugui Island (F5) C-22

Table C-8 Strontium-90 radionuclide concentration summary for all soil profiles taken during the 1978 NMIRS on Lomiulal Island (F7)...................... -23

Table C-9 Strontium-90 radionuclide concentration summary for all soil profiles taken during the 1978 NMIRS on Kabelle Island (F13)....................... -24

Table C-10 Strontium-90 radionuclide concentration summary of all soil profiles taken during the 1978 NMIRS on Mellu Island (F23). C-25 
Table C-11 Plutonium 239+240 radionuclide concentration summary for all soil profiles taken during the 1978 NMIRS together with our recent trip in 1989 on Naen Island (F1). C-26

Table C-12 Plutonium 239+240 radionuclide concentration summary for all soil profiles taken during the 1978 NMIRS together with our recent trip in 1989 on Yugui Island (F5).

Table C-13 Plutonium $239+240$ radionuclide concentration summary for all soil profiles taken during the 1978 NMIRS together with our recent trip in 1989 on Lomiulal Island (F7). C-28

Table C-14 Plutonium 239+240 radionuclide concentration summary for all soil profiles taken during the 1978 NMIRS on Kabelle Island (F13).......C-29

Table C-15 Plutonium $239+240$ radionuclide concentration summary for all soil profiles taken during the 1978 NMIRS together with our recent trip in 1989 on Mellu Island (F23). C-30

Table C-16 Americium-241 radionuclide concentration summary for all soil profiles taken from the 1978 NMIRS together with our recent trip in 1989 on Naen Island (F1).

Table C-17 Americium-241 radionuclide concentration summary for all soil profiles taken from the 1978 NMIRS together with our most recent trip in 1989 on Yugui Island (F5). .C-32

Table C-18 Americium-241 radionuclide concentration summary for all soil profiles taken from the 1978 NMIRS together with our most recent trip in 1989 on Lomiulal Island (F7). .C-33

Table C-19 Americium-241 radionuclide concentration summary for all soil profiles taken from the 1978 NMIRS together with our most recent trips in 1988 through 1989 on Kabelle Island (F13)..

Table C-20 Americium-241 radionuclide concentration summary for all soil profiles taken from the 1978 NMIRS together with our most recent trip in 1989 on Mellu Island (F23)...................................................................35

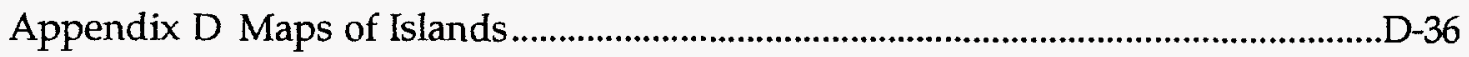




\section{INTRODUCTORY COMMENTS}

The data presented in the following tables is the total available for each northern island; they include both the data from the 1978 Northern Marshall Island Radiological Survey (NMIRS) and trips to Rongelap Atoll from 1986 through 1989.

In one table we present the number of vegetation samples collected in the 1978 NMIRS and from 1986 through 1989. Again the majority of the ${ }^{137} \mathrm{Cs}$ data is from the 1986-1989 trips. We have not made additional analyses of $239+240 \mathrm{Pu}$, ${ }^{241} \mathrm{Am}$ and ${ }^{90} \mathrm{Sr}$ because the concentrations are very low and these radionuclides contribute less than $5 \%$ of an already very small dose.

In another table we show the number of soil samples collected at each island in 1978 and the number collected since 1986. Most of the data are from 1986 through 1989. The major exception is ${ }^{90} \mathrm{Sr}$ where all of the data are from the 1978 NMIRS. We have done some additional $\mathrm{Pu}$ analyses of surface soils $(0-5 \mathrm{~cm}$ depth) in the northern islands. A significant amount of new data for ${ }^{137} \mathrm{Cs}$ and ${ }^{241} \mathrm{Am}$ have been generated from the samples collected from 1986 through 1989.

The data are presented in the form of summary tables, graphics, detailed appendices and aerial photographs of the islands with the sample locations marked. The identified sample locations from the 1978 NMIRS will be added later. 
Table 1. The number of food crops collected at the northern islands at Rongelap Atoll and the mean and median ${ }^{137} \mathrm{Cs}$ concentrations.

\begin{tabular}{|c|c|c|c|c|c|c|}
\hline \multirow{2}{*}{$\begin{array}{l}\text { Island } \\
\text { Name }\end{array}$} & \multirow{2}{*}{$\begin{array}{l}\text { Island } \\
\text { Code }\end{array}$} & \multicolumn{3}{|c|}{ Number of Samples } & \multicolumn{2}{|c|}{ Cs-137pCi/g wet wt. } \\
\hline & & Total & 1978 NMIRS & 1986-1989 & median & mean \\
\hline \multicolumn{7}{|c|}{ Drinking Coconut Meat } \\
\hline Naen & $01 f$ & 8 & 2 & 6 & 4.2 & 6.7 \\
\hline Lomiulal & $07 \mathrm{f}$ & 1 & 1 & 0 & 9.9 & 9.9 \\
\hline Kabelle & $13 \mathrm{f}$ & 29 & 1 & 28 & 1.9 & 2.2 \\
\hline Mellu & $23 f$ & 1 & 0 & 1 & 3.6 & 3.6 \\
\hline \multicolumn{7}{|c|}{ Drinking Coconut Juice } \\
\hline Naen & $01 \mathrm{f}$ & 6 & 0 & 6 & 1.5 & 3.3 \\
\hline Lomiulal & $07 f$ & 1 & 1 & 0 & 1.4 & 1.4 \\
\hline Kabelle & $13 \mathrm{f}$ & 28 & 1 & 27 & 0.86 & 1.0 \\
\hline Mellu & $23 f$ & 1 & 0 & 1 & 2.7 & 2.7 \\
\hline \multicolumn{7}{|c|}{ Copra Meat } \\
\hline Naen & 01f & 5 & 2 & 3 & 7.7 & 7.5 \\
\hline Yugui & $05 f$ & 6 & 0 & 6 & 12 & 12 \\
\hline Lomiulal & $07 f$ & 8 & 2 & 6 & 11 & 12 \\
\hline Kabelle & $13 f$ & 23 & 2 & 21 & 3.3 & 4.4 \\
\hline Mellu & $23 \mathrm{f}$ & 12 & 3 & 9 & 2.8 & 4.0 \\
\hline \multicolumn{7}{|c|}{ Copra Juice } \\
\hline Naen & $01 f$ & 5 & 2 & 3 & 3.2 & 5.8 \\
\hline Yugui & $05 f$ & 6 & 0 & 6 & 10 & 8.6 \\
\hline Lomiulal & $07 \mathrm{f}$ & 8 & 2 & 6 & 7.2 & 7.3 \\
\hline Kabelle & $13 f$ & 22 & 2 & 20 & 2.4 & 2.5 \\
\hline Mellu & $23 \mathrm{f}$ & 11 & 2 & 9 & 2.1 & 3.1 \\
\hline \multicolumn{7}{|c|}{ Pandanus } \\
\hline Naen & $01 \mathrm{f}$ & 4 & 3 & 1 & 5.7 & 6.1 \\
\hline Yugui & $05 \mathrm{f}$ & 6 & 2 & 4 & 8.2 & 8.3 \\
\hline Kabelle & $13 \mathrm{f}$ & 2 & 0 & 2 & 7.3 & 7.3 \\
\hline Mellu & $23 \mathrm{f}$ & 8 & 4 & 4 & 3.4 & 4.2 \\
\hline \multicolumn{7}{|c|}{ Tacca Meat } \\
\hline Yugui & $05 \mathrm{f}$ & 2 & 0 & 2 & 10 & 10 \\
\hline Lomiulal & $07 f$ & 1 & 1 & 0 & 1.3 & 1.3 \\
\hline Mellu & 231 & 2 & 1 & 1 & 2.4 & 2.4 \\
\hline
\end{tabular}

NOTE: Specific activity is decay corrected to 1994. 
Table 2. The number of indicator species collected at the northern islands at Rongelap Atoll and the mean and median ${ }^{137} \mathrm{Cs}$ concentrations.

\begin{tabular}{|c|c|c|c|c|c|c|}
\hline \multirow{2}{*}{$\begin{array}{l}\text { Island } \\
\text { Name }\end{array}$} & \multirow{2}{*}{$\begin{array}{l}\text { Island } \\
\text { Code }\end{array}$} & \multicolumn{3}{|c|}{ Number of Samples } & \multicolumn{2}{|c|}{ Cs-137 pCi/g wet wt. } \\
\hline & & Total & 1978 NMIRS & $1986-1989$ & median & mean \\
\hline \multicolumn{7}{|c|}{ Morinda Fruit } \\
\hline Kabelle & $13 f$ & 1 & 1 & 0 & 1.2 & 1.2 \\
\hline \multicolumn{7}{|c|}{ Guettardia Leaves } \\
\hline Kabelle & $13 f$ & 7 & 0 & 7 & 0.45 & 0.43 \\
\hline \multicolumn{7}{|c|}{ Messerschmidia Leaves } \\
\hline Naen & $01 f$ & 2 & 2 & 0 & 8.7 & 8.7 \\
\hline Kabelle & $13 f$ & 11 & 0 & 11 & 1.2 & 1.3 \\
\hline \multicolumn{7}{|c|}{ Pisonia Leaves } \\
\hline Naen & $01 f$ & 1 & 0 & 1 & 15 & 15 \\
\hline Yugui & $05 f$ & 1 & 0 & 1 & 15 & 15 \\
\hline Lomiulal & $07 \mathrm{f}$ & 5 & 1 & 4 & 8.9 & 8.3 \\
\hline Kabelle & $13 f$ & 4 & 0 & 4 & 4.8 & 4.6 \\
\hline \multicolumn{7}{|c|}{ Pandanus Leaves } \\
\hline Yugui & $05 f$ & 1 & 1 & 0 & 12 & 12 \\
\hline Lomiulal & $07 f$ & 1 & 1 & 0 & 8.3 & 8.3 \\
\hline Kabelle & $13 f$ & 1 & 0 & 1 & 2.4 & 2.4 \\
\hline \multicolumn{7}{|c|}{ Scaevola Leaves } \\
\hline Naen & $01 \mathrm{f}$ & 6 & 1 & 5 & 1.6 & 1.8 \\
\hline Yugui & $05 f$ & 5 & 0 & 5 & 2.0 & 2.2 \\
\hline Lomiulal & $07 f$ & 9 & 0 & 9 & 1.3 & 1.6 \\
\hline Kabelle & $13 \mathrm{f}$ & 16 & 0 & 16 & 1.0 & 1.6 \\
\hline \multicolumn{7}{|c|}{ Coconut Fronds } \\
\hline Kabelle & $13 \mathrm{f}$ & 8 & 0 & 8 & 3.0 & 3.0 \\
\hline
\end{tabular}

NOTE: Specific activity decay corrected to 1994. 
Table 3. The number of animal samples collected at the northern islands at Rongelap Atoll and the mean and median ${ }^{137} \mathrm{Cs}$ concentrations.

\begin{tabular}{|c|c|c|c|c|c|c|}
\hline \multirow{2}{*}{$\begin{array}{l}\text { Island } \\
\text { Name } \\
\end{array}$} & \multirow{2}{*}{$\begin{array}{l}\text { Island } \\
\text { Code }\end{array}$} & \multicolumn{3}{|c|}{ Number of Samples } & \multicolumn{2}{|c|}{ Cs-137 pCi/g wet wt. } \\
\hline & & Total & 1978 NMIRS & 1986-1989 & median & mean \\
\hline \multicolumn{7}{|c|}{ Coconut Crab Muscle } \\
\hline Naen & $01 f$ & 1 & 0 & 1 & 14 & 14 \\
\hline Yugui & $05 \mathrm{f}$ & 1 & 0 & 1 & 20 & 20 \\
\hline Loniulal & $07 f$ & 2 & 1 & 1 & 10 & 10 \\
\hline Kabelle & $13 \mathrm{f}$ & 10 & 0 & 10 & 4.0 & 5.2 \\
\hline Mellu & $23 \mathrm{f}$ & 3 & 1 & 2 & 3.5 & 3.9 \\
\hline \multicolumn{7}{|c|}{ Coconut Crab Hepatopancreas } \\
\hline Naen & $01 f$ & 1 & 0 & 1 & 14 & 14 \\
\hline Yugui & $05 f$ & 1 & 0 & 1 & 17 & 17 \\
\hline Loniulal & $07 f$ & 1 & 0 & 1 & 7.0 & 7.0 \\
\hline Kabelle & $13 f$ & 9 & 0 & 9 & 5.7 & 7.6 \\
\hline Mellu & $23 \mathrm{f}$ & 2 & 0 & 2 & 3.2 & 3.2 \\
\hline
\end{tabular}

NOTE: Specific activity decay corrected to 1994. 
Table 4. The number of soil samples collected at the norhtern islands at Rongelap Atoll.

\begin{tabular}{|c|c|c|c|c|c|}
\hline \multirow{3}{*}{$\begin{array}{l}\text { Island } \\
\text { Name } \\
\end{array}$} & \multirow{3}{*}{$\begin{array}{r}\text { Island } \\
\text { Code }\end{array}$} & \multicolumn{4}{|c|}{ Number of Soil Samples for Cs-137 and Am-241 } \\
\hline & & \multicolumn{2}{|c|}{1978} & \multicolumn{2}{|c|}{ 1986-1989 } \\
\hline & & Profiles & $0-5 \mathrm{~cm}$. & Profiles & $0-5 \mathrm{~cm}$. \\
\hline Naen & $01 f$ & 7 & 6 & 10 & 10 \\
\hline Yugui & $05 f$ & 1 & 1 & 10 & 10 \\
\hline Lomiulal & $07 f$ & 4 & 4 & 10 & 10 \\
\hline Kabelle & $13 f$ & 5 & 5 & 12 & 29 \\
\hline \multirow[t]{2}{*}{ Mellu } & $23 \mathrm{f}$ & 4 & 4 & 11 & 11 \\
\hline & & \multicolumn{4}{|c|}{ Number of Soil Samples for Pu- $239+240$} \\
\hline Island & Island & \multicolumn{2}{|c|}{1978} & \multicolumn{2}{|c|}{$1986-1989$} \\
\hline Name & Code & Profiles & $0-5 \mathrm{~cm}$ & Profiles & $0-5 \mathrm{~cm}$. \\
\hline Naen & $01 f$ & 7 & 7 & 0 & 9 \\
\hline Yugui & $05 f$ & 1 & 1 & 0 & 10 \\
\hline Lomiulal & $07 f$ & 4 & 4 & 0 & 10 \\
\hline Kabelle & $13 f$ & 5 & 5 & 0 & 0 \\
\hline \multirow[t]{2}{*}{ Mellu } & $23 f$ & 4 & 4 & 0 & 11 \\
\hline & & \multicolumn{4}{|c|}{ Number of Soil Samples for Sr-90 } \\
\hline \multirow{2}{*}{$\begin{array}{l}\text { Island } \\
\text { Name } \\
\end{array}$} & \multirow{2}{*}{$\begin{array}{r}\text { Island } \\
\text { Code } \\
\end{array}$} & \multicolumn{2}{|c|}{1978} & \multicolumn{2}{|c|}{ 1986-1989 } \\
\hline & & Profiles & $0-5 \mathrm{~cm}$. & Profiles & $0-5 \mathrm{~cm}$. \\
\hline Naen & $01 f$ & 7 & 7 & 0 & 0 \\
\hline Yugui & $05 \mathrm{f}$ & 1 & 1 & 0 & 0 \\
\hline Lomiulal & $07 \mathrm{f}$ & 4 & 4 & 0 & 0 \\
\hline Kabelle & $13 \mathrm{f}$ & 5 & 5 & 0 & 0 \\
\hline Mellu & $23 f$ & 4 & 4 & 0 & 0 \\
\hline
\end{tabular}


Table 5. Average ${ }^{137} \mathrm{Cs},{ }^{90} \mathrm{Sr}_{,}{ }^{239+240} \mathrm{Pu}$, and ${ }^{241} \mathrm{Am}$ soil concentrations in $\mathrm{pCi} / \mathrm{g}$ dry weight for soil profiles taken on Naen Island (F1).

\begin{tabular}{|c|c|c|c|c|c|c|c|c|}
\hline \multirow{2}{*}{$\begin{array}{l}\text { Soil } \\
\text { depth } \\
(\mathrm{cm})\end{array}$} & \multicolumn{2}{|c|}{${ }^{137} \mathrm{Cs}$} & \multicolumn{2}{|c|}{${ }^{90} \mathrm{Sr}$} & \multicolumn{2}{|c|}{$239+240 \mathrm{Pu}$} & \multicolumn{2}{|c|}{${ }^{241} \mathrm{Am}$} \\
\hline & Median & Mean & Median & Mean & Median & Mean & Median & Mean \\
\hline $00-05$ & 83 & 120 & 110 & 110 & 49 & 43 & 26 & 29 \\
\hline $05-10$ & 44 & 46 & 77 & 79 & 18 & 21 & 8.7 & 8.5 \\
\hline $10-15$ & 21 & 23 & 41 & 51 & 8.2 & 11 & 3.3 & 3.3 \\
\hline $15-25$ & 7.7 & 7.1 & 10 & 15 & 1.4 & 2.2 & 0.63 & 1.1 \\
\hline $25-40$ & 1.5 & 1.9 & 6.9 & 9.7 & 0.14 & 0.69 & 0.12 & 0.31 \\
\hline $40-60$ & 1.5 & 1.7 & 3.6 & 3.7 & 0.18 & 0.20 & 0.069 & 0.11 \\
\hline $00-05$ & 83 & 120 & 110 & 110 & 49 & 43 & 26 & 29 \\
\hline $00-10$ & 69 & 84 & 87 & 93 & 23 & 25 & 21 & 19 \\
\hline $00-15$ & 53 & 64 & 70 & 79 & 18 & 20 & 12 & 13 \\
\hline $00-25$ & 36 & 41 & 47 & 53 & 11 & 13 & 6.8 & 7.1 \\
\hline $00-40$ & 24 & 26 & 32 & 37 & 6.8 & 8.4 & 4.3 & 4.6 \\
\hline $00-60$ & 13 & 14 & 23 & 26 & 4.5 & 5.7 & 2.4 & 2.5 \\
\hline
\end{tabular}

NOTE: Specific activity decay corrected to 1994.

Table 6. Average ${ }^{137} \mathrm{Cs},{ }^{90} \mathrm{Sr},{ }^{239+240} \mathrm{Pu}$, and ${ }^{241} \mathrm{Am}$ soil concentrations in $\mathrm{pCi} / \mathrm{g}$ dry weight for soil profiles taken on $\mathrm{Yugui}$ Island (F5).

\begin{tabular}{|c|c|c|c|c|c|c|c|c|}
\hline \multirow{2}{*}{$\begin{array}{l}\text { Soil } \\
\text { depth } \\
\text { (cm) }\end{array}$} & \multicolumn{2}{|c|}{${ }^{137} \mathrm{Cs}$} & \multicolumn{2}{|c|}{${ }^{90} \mathrm{Sr}$} & \multicolumn{2}{|c|}{$239+240 \mathrm{Pu}$} & \multicolumn{2}{|c|}{${ }^{241} \mathrm{Am}$} \\
\hline & Median & Mean & Median & Mean & Median & $\overline{\text { Mean }}$ & Median & Mean \\
\hline $00-05$ & 170 & 170 & 32 & 32 & 55 & 58 & 48 & 53 \\
\hline $05-10$ & 73 & 86 & 20 & 20 & 2.3 & 2.3 & 4.3 & 16 \\
\hline $10-15$ & 32 & 28 & 11 & 11 & 0.66 & 0.66 & 0.89 & 1.7 \\
\hline $15-25$ & 8.8 & 9.7 & 3.6 & 3.6 & 0.23 & 0.23 & 0.32 & 0.40 \\
\hline $25-40$ & 2.8 & 3.2 & 2.7 & 2.7 & 0.12 & 0.12 & 0.054 & 0.054 \\
\hline $40-60$ & 0.53 & 0.53 & 0.21 & 0.21 & 0.032 & 0.032 & 0.0 & 0.0 \\
\hline $00-05$ & 170 & 170 & 32 & 32 & 55 & 58 & 48 & 53 \\
\hline $00-10$ & 140 & 130 & 26 & 26 & 8.6 & 8.6 & 27 & 34 \\
\hline $00-15$ & 100 & 95 & 21 & 21 & 5.9 & 5.9 & 19 & 21 \\
\hline $00-25$ & 65 & 61 & 14 & 14 & 3.7 & 3.7 & 18 & 18 \\
\hline $00-40$ & 42 & 39 & 9.9 & 9.9 & 2.3 & 2.3 & 1.1 & 1.1 \\
\hline $00-60$ & 14 & 14 & 6.7 & 6.7 & 1.6 & 1.6 & 0.0 & 0.0 \\
\hline
\end{tabular}

NOTE: Specific activity decay corrected to 1994. 
Table 7. Average ${ }^{137} \mathrm{Cs},{ }^{90} \mathrm{Sr}^{239+240} \mathrm{Pu}$, and ${ }^{241} \mathrm{Am}$ soil concentrations in $\mathrm{pCi} / \mathrm{g}$ dry weight for soil profiles taken on Lomiulal Island (F).

\begin{tabular}{|c|c|c|c|c|c|c|c|c|}
\hline \multirow{2}{*}{$\begin{array}{l}\text { Soil } \\
\text { depth } \\
\text { (cm) }\end{array}$} & \multicolumn{2}{|c|}{${ }^{137} \mathrm{Cs}$} & \multicolumn{2}{|c|}{${ }^{90} \mathrm{Sr}$} & \multicolumn{2}{|c|}{$239+240 \mathrm{Pu}$} & \multicolumn{2}{|c|}{${ }^{241} \mathrm{Am}$} \\
\hline & Median & Mean & Median & Mean & Median & $\overline{\text { Mean }}$ & Median & Mean \\
\hline $00-05$ & 53 & 81 & 51 & 66 & 35 & 34 & 22 & 22 \\
\hline $05-10$ & 37 & 35 & 49 & 46 & 16 & 13 & 4.9 & 6.3 \\
\hline $10-15$ & 15 & 18 & 26 & 25 & 4.4 & 6.3 & 2.3 & 3.0 \\
\hline $15-25$ & 6.3 & 9.4 & 12 & 16 & 1.1 & 2.3 & 0.37 & 0.84 \\
\hline $25-40$ & 1.5 & 3.2 & 6.3 & 7.8 & 0.41 & 0.41 & 0.19 & 0.18 \\
\hline $40-60$ & 0.39 & 1.1 & 2.6 & 3.4 & 0.13 & 0.23 & 0.18 & 0.18 \\
\hline $00-05$ & 53 & 81 & 51 & 66 & 35 & 34 & 22 & 22 \\
\hline $00-10$ & 47 & 58 & 45 & 56 & 16 & 16 & 13 & 14 \\
\hline $00-15$ & 39 & 44 & 37 & 46 & 13 & 13 & 8.1 & 9.3 \\
\hline $00-25$ & 30 & 30 & 31 & 34 & 8.4 & 8.6 & 5.5 & 5.7 \\
\hline $00-40$ & 21 & 20 & 24 & 24 & 5.4 & 5.5 & 3.1 & 3.1 \\
\hline $00-60$ & 9.5 & 8.9 & 17 & 17 & 3.7 & 3.8 & 2.1 & 2.1 \\
\hline
\end{tabular}

NOTE: Specific activity decay corrected to 1994.

V

Table 8. Average ${ }^{137} \mathrm{Cs},{ }^{90} \mathrm{Sr},{ }^{239+240} \mathrm{Pu}$, and ${ }^{241} \mathrm{Am}$ soil concentrations in $\mathrm{pCi} / \mathrm{g}$ dry weight for soil profiles taken on Kabelle Island (F13).

\begin{tabular}{|c|c|c|c|c|c|c|c|c|}
\hline \multirow{2}{*}{$\begin{array}{l}\text { Soil } \\
\text { depth } \\
\text { (cm) }\end{array}$} & \multicolumn{2}{|c|}{${ }^{137} \mathrm{Cs}$} & \multicolumn{2}{|c|}{${ }^{90} \mathrm{Sr}$} & \multicolumn{2}{|c|}{$239+240 \mathrm{Pu}$} & \multicolumn{2}{|c|}{${ }^{241} \mathrm{Am}$} \\
\hline & Median & Mean & Median & Mean & Median & Mean & Median & Mean \\
\hline $00-05$ & 28 & 34 & 26 & 32 & 8.3 & 14 & 11 & 12 \\
\hline $05-10$ & 14 & 15 & 7.2 & 12 & 1.6 & 3.1 & 3.7 & 4.9 \\
\hline $10-15$ & 7.6 & 9.4 & 8.6 & 16 & 0.68 & 3.6 & 0.79 & 1.9 \\
\hline $15-25$ & 3.2 & 6.2 & 4.6 & 12 & 1.7 & 2.9 & 0.98 & 1.0 \\
\hline $25-40$ & 1.4 & 2.1 & 3.7 & 3.8 & 0.36 & 0.71 & 0.49 & 0.48 \\
\hline $40-60$ & 0.42 & 0.48 & 2.6 & 2.2 & 0.12 & 0.23 & 0.12 & 0.12 \\
\hline $00-05$ & 28 & 34 & 26 & 32 & 8.3 & 14 & 11 & 12 \\
\hline $00-10$ & 19 & 22 & 20 & 22 & 8.0 & 8.7 & 7.0 & 8.2 \\
\hline $00-15$ & 18 & 18 & 20 & 20 & 6.8 & 7.0 & 5.0 & 6.0 \\
\hline $00-25$ & 14 & 13 & 16 & 17 & 6.7 & 5.3 & 3.6 & 3.3 \\
\hline $00-40$ & 9.2 & 8.8 & 10 & 12 & 4.4 & 3.6 & 2.1 & 1.9 \\
\hline $00-60$ & 5.6 & 5.9 & 7.2 & 8.7 & 3.0 & 2.5 & 0.87 & 0.87 \\
\hline
\end{tabular}

NOTE: Specific activity decay corrected to 1994. 
Table 9. Average ${ }^{137} \mathrm{Cs},{ }^{90} \mathrm{Sr},{ }^{239+240} \mathrm{Pu}$, and ${ }^{241} \mathrm{Am}$ soil concentrations in $\mathrm{pCi} / \mathrm{g}$ dry weight for soil profiles taken on Mellu Island (F23).

\begin{tabular}{|c|c|c|c|c|c|c|c|c|}
\hline \multirow{2}{*}{$\begin{array}{l}\text { Soil } \\
\text { depth } \\
\text { (cm) }\end{array}$} & \multicolumn{2}{|c|}{${ }^{137} \mathrm{Cs}$} & \multicolumn{2}{|c|}{${ }^{90} \mathrm{Sr}$} & \multicolumn{2}{|c|}{${ }^{239}+240 \mathrm{Pu}$} & \multicolumn{2}{|c|}{${ }^{241} \mathrm{Am}$} \\
\hline & Median & Mean & Median & $\overline{\text { Mean }}$ & Median & Mean & Median & Mean \\
\hline $00-05$ & 32 & 38 & 35 & 32 & 17 & 14 & 7.5 & 9.2 \\
\hline $05-10$ & 19 & 29 & 27 & 23 & 3.5 & 5.3 & 3.4 & 5.6 \\
\hline $10-15$ & 13 & 17 & 14 & 17 & 2.6 & 3.6 & 2.3 & 3.3 \\
\hline $15-25$ & 9.9 & 11 & 12 & 13 & 1.3 & 1.5 & 1.6 & 1.7 \\
\hline $25-40$ & 4.0 & 5.0 & 10 & 8.3 & 1.1 & 1.1 & 0.42 & 0.47 \\
\hline $40-60$ & 2.0 & 2.0 & 2.3 & 2.3 & 0.064 & 0.064 & 0.024 & 0.024 \\
\hline 00-05 & 32 & 38 & 35 & 32 & 17 & 14 & 7.5 & 9.2 \\
\hline $00-10$ & 27 & 34 & 31 & 27 & 9.9 & 9.9 & 7.6 & 7.6 \\
\hline $00-15$ & 22 & 28 & 25 & 24 & 7.0 & 7.8 & 6.8 & 6.5 \\
\hline $00-25$ & 16 & 21 & 19 & 20 & 4.4 & 5.3 & 4.5 & 4.8 \\
\hline $00-40$ & 13 & 16 & 14 & 15 & 3.0 & 3.7 & 1.7 & 2.1 \\
\hline $00-60$ & 21 & 21 & 12 & 12 & 1.8 & 1.8 & 0.94 & 0.94 \\
\hline
\end{tabular}

NOTE: Specific activity decay corrected to 1994. 


\section{${ }^{137} \mathrm{Cs}$ in Soil at Rongelap Atoll--Northern Islands}

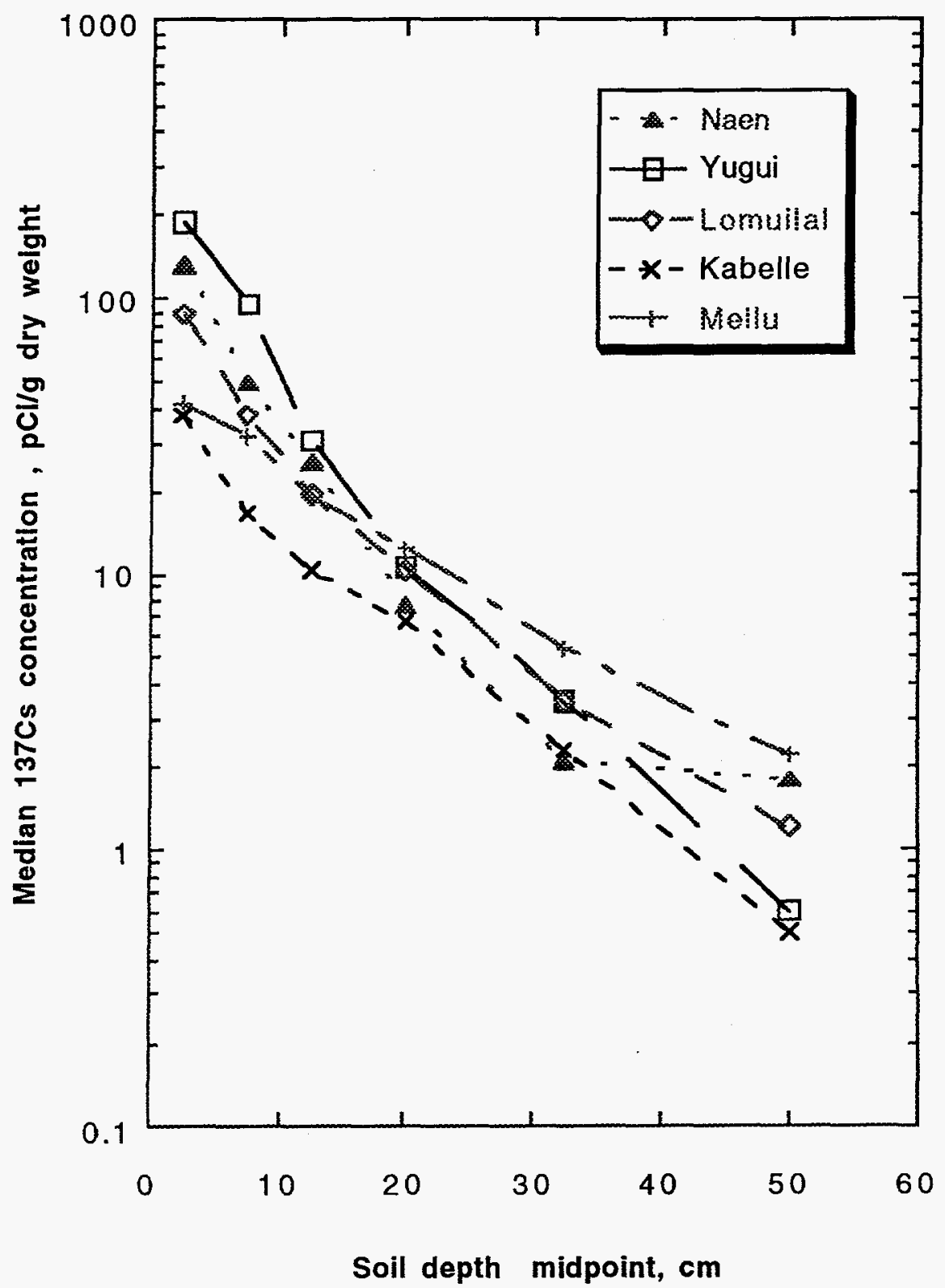

Figure $1 .{ }^{137} \mathrm{Cs}$ in soil at rongelap Atoll-northern islands. 
Appendix A

Radionuclide concentration summary in vegetation samples collected during the 1978 NMIRS and from 1986 through 1989 in the northern islands of Rongelap Atoll. 
Table A-1. The ${ }^{137} \mathrm{Cs}$ radionuclide concentration summary for vegetation (food crops) collected from the northern islands of Rongelap Atoll during the 1978 NMIRS together with our recent trips in 1986 through 1989.

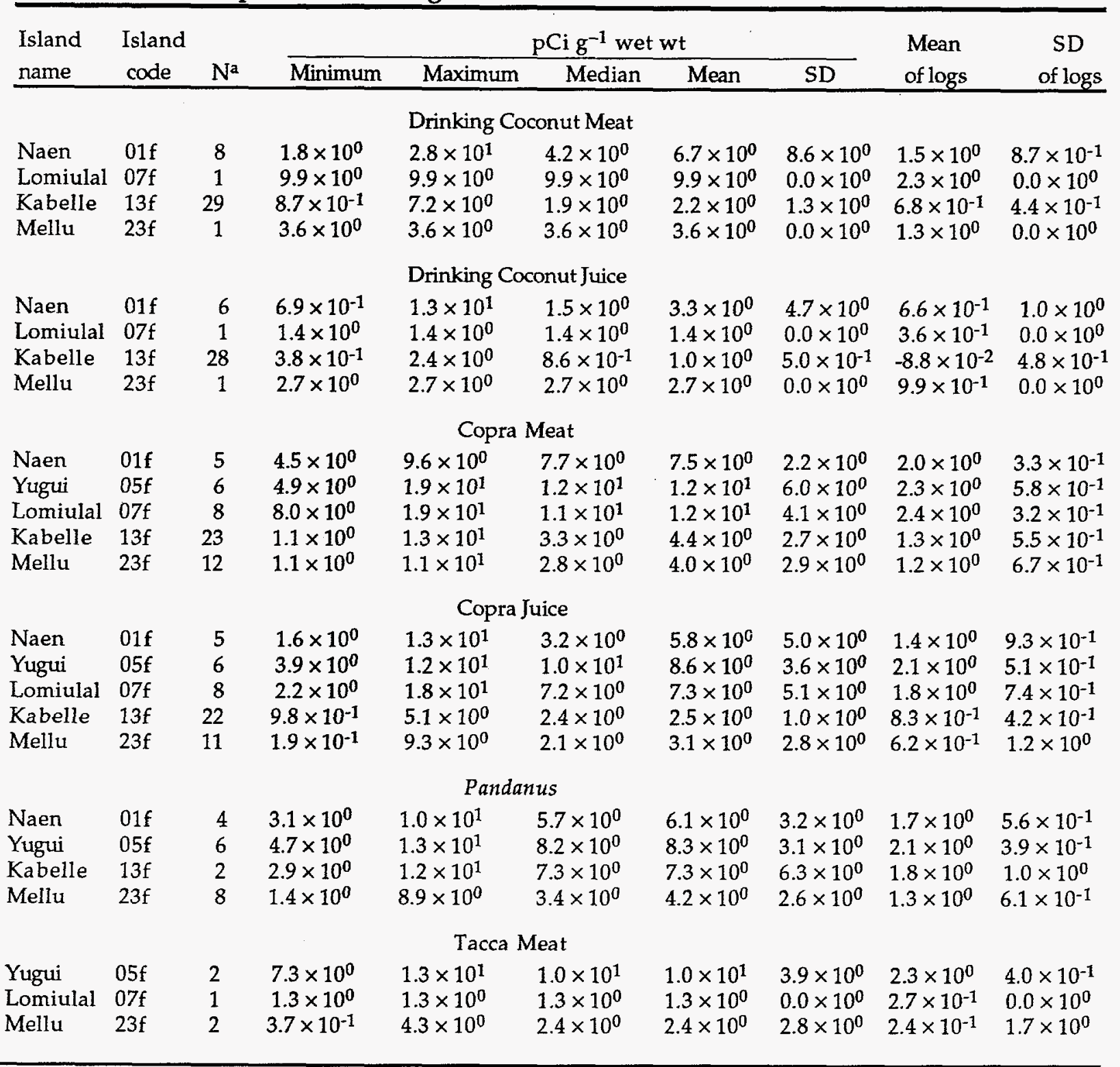

NOTE: Specific activity is decay corrected to 1994. $\mathrm{Na}^{\mathrm{a}}$ stands for number of individual samples. 
Table A-2. The ${ }^{137} \mathrm{Cs}$ radionuclide concentration summary for vegetation (indicator species) collected from the northern islands of Rongelap Atoll during the 1978 NMIRS together with our recent trips in 1986 through 1989.

\begin{tabular}{|c|c|c|c|c|c|c|c|c|c|}
\hline \multirow{2}{*}{$\begin{array}{l}\text { Island } \\
\text { name }\end{array}$} & \multirow{2}{*}{$\begin{array}{l}\text { Island } \\
\text { code } \\
\end{array}$} & \multirow[b]{2}{*}{$\mathrm{N}^{\mathrm{a}}$} & \multicolumn{5}{|c|}{ pCi $g^{-1}$ wet wt } & \multirow{2}{*}{$\begin{array}{l}\text { Mean } \\
\text { of logs }\end{array}$} & \multirow{2}{*}{$\begin{array}{c}\mathrm{SD} \\
\text { of logs } \\
\end{array}$} \\
\hline & & & Minimum & Maximum & Median & Mean & SD & & \\
\hline \multicolumn{10}{|c|}{ Morinda Fruit } \\
\hline Kabelle & $13 \mathrm{f}$ & 1 & $1.2 \times 10^{0}$ & $1.2 \times 10^{0}$ & $1.2 \times 10^{0}$ & $1.2 \times 10^{0}$ & $0.0 \times 10^{0}$ & $1.5 \times 10^{-1}$ & $0.0 \times 10^{0}$ \\
\hline \multicolumn{10}{|c|}{ Guettardia Leaves } \\
\hline Kabelle & $13 f$ & 7 & $1.9 \times 10^{-1}$ & $6.2 \times 10^{-1}$ & $4.5 \times 10^{-1}$ & $4.3 \times 10^{-1}$ & $1.5 \times 10^{-1}$ & $-9.1 \times 10^{-1}$ & $4.0 \times 10^{-1}$ \\
\hline \multicolumn{10}{|c|}{ Messerschmidia Leaves } \\
\hline $\begin{array}{l}\text { Naen } \\
\text { Kabelle }\end{array}$ & $\begin{array}{l}01 \mathrm{f} \\
13 \mathrm{f}\end{array}$ & $\begin{array}{r}2 \\
11\end{array}$ & $\begin{array}{l}7.4 \times 10^{0} \\
3.3 \times 10^{-2}\end{array}$ & $\begin{array}{l}9.9 \times 10^{0} \\
2.2 \times 10^{0}\end{array}$ & $\begin{array}{l}8.7 \times 10^{0} \\
1.2 \times 10^{0}\end{array}$ & $\begin{array}{l}8.7 \times 10^{0} \\
1.3 \times 10^{0}\end{array}$ & $\begin{array}{l}1.7 \times 10^{0} \\
7.4 \times 10^{-1}\end{array}$ & $\begin{array}{c}2.2 \times 10^{0} \\
-2.7 \times 10^{-1}\end{array}$ & $\begin{array}{l}2.0 \times 10^{-1} \\
1.5 \times 10^{0}\end{array}$ \\
\hline \multicolumn{10}{|c|}{ Pisonia Leaves } \\
\hline $\begin{array}{l}\text { Naen } \\
\text { Yugui } \\
\text { Lomiulal } \\
\text { Kabelle }\end{array}$ & $\begin{array}{l}01 f \\
05 f \\
07 f \\
13 f\end{array}$ & $\begin{array}{l}1 \\
1 \\
5 \\
4\end{array}$ & $\begin{array}{l}1.5 \times 10^{1} \\
1.5 \times 10^{1} \\
4.6 \times 10^{0} \\
2.5 \times 10^{0}\end{array}$ & $\begin{array}{l}1.5 \times 10^{1} \\
1.5 \times 10^{1} \\
1.0 \times 10^{1} \\
6.3 \times 10^{0}\end{array}$ & $\begin{array}{l}1.5 \times 10^{1} \\
1.5 \times 10^{1} \\
8.9 \times 10^{0} \\
4.8 \times 10^{0}\end{array}$ & $\begin{array}{l}1.5 \times 10^{1} \\
1.5 \times 10^{1} \\
8.3 \times 10^{0} \\
4.6 \times 10^{0}\end{array}$ & $\begin{array}{l}0.0 \times 10^{0} \\
0.0 \times 10^{0} \\
2.2 \times 10^{0} \\
1.6 \times 10^{0}\end{array}$ & $\begin{array}{l}2.7 \times 10^{0} \\
2.7 \times 10^{0} \\
2.1 \times 10^{0} \\
1.5 \times 10^{0}\end{array}$ & $\begin{array}{l}0.0 \times 10^{0} \\
0.0 \times 10^{0} \\
3.1 \times 10^{-1} \\
3.9 \times 10^{-1}\end{array}$ \\
\hline \multicolumn{10}{|c|}{ Pandanus Leaves } \\
\hline $\begin{array}{l}\text { Yugui } \\
\text { Lomiulal } \\
\text { Kabelle }\end{array}$ & $\begin{array}{l}05 f \\
07 f \\
13 f\end{array}$ & $\begin{array}{l}1 \\
1 \\
1\end{array}$ & $\begin{array}{l}1.2 \times 10^{1} \\
8.3 \times 10^{0} \\
2.4 \times 10^{0}\end{array}$ & $\begin{array}{l}1.2 \times 10^{1} \\
8.3 \times 10^{0} \\
2.4 \times 10^{0}\end{array}$ & $\begin{array}{l}1.2 \times 10^{1} \\
8.3 \times 10^{0} \\
2.4 \times 10^{0}\end{array}$ & $\begin{array}{l}1.2 \times 10^{1} \\
8.3 \times 10^{0} \\
2.4 \times 10^{0}\end{array}$ & $\begin{array}{l}0.0 \times 10^{0} \\
0.0 \times 10^{0} \\
0.0 \times 10^{0}\end{array}$ & $\begin{array}{l}2.5 \times 10^{0} \\
2.1 \times 10^{0} \\
8.9 \times 10^{-1}\end{array}$ & $\begin{array}{l}0.0 \times 10^{0} \\
0.0 \times 10^{0} \\
0.0 \times 10^{0}\end{array}$ \\
\hline \multicolumn{10}{|c|}{ Scaevola Leaves } \\
\hline $\begin{array}{l}\text { Naen } \\
\text { Yugui } \\
\text { Lomiulal } \\
\text { Kabelle }\end{array}$ & $\begin{array}{l}01 f \\
05 f \\
07 f \\
13 f\end{array}$ & $\begin{array}{r}6 \\
5 \\
9 \\
16\end{array}$ & $\begin{array}{l}7.4 \times 10^{-1} \\
5.9 \times 10^{-1} \\
8.6 \times 10^{-1} \\
5.3 \times 10^{-1}\end{array}$ & $\begin{array}{l}3.2 \times 10^{0} \\
4.3 \times 10^{0} \\
3.4 \times 10^{0} \\
5.2 \times 10^{0}\end{array}$ & $\begin{array}{l}1.6 \times 10^{0} \\
2.0 \times 10^{0} \\
1.3 \times 10^{0} \\
1.0 \times 10^{0}\end{array}$ & $\begin{array}{l}1.8 \times 10^{0} \\
2.2 \times 10^{0} \\
1.6 \times 10^{0} \\
1.6 \times 10^{0}\end{array}$ & $\begin{array}{c}1.1 \times 10^{0} \\
1.4 \times 10^{0} \\
8.1 \times 10^{-1} \\
1.4 \times 10^{0}\end{array}$ & $\begin{array}{l}4.4 \times 10^{-1} \\
5.8 \times 10^{-1} \\
3.5 \times 10^{-1} \\
1.9 \times 10^{-1}\end{array}$ & $\begin{array}{l}6.5 \times 10^{-1} \\
7.4 \times 10^{-1} \\
4.6 \times 10^{-1} \\
7.0 \times 10^{-1}\end{array}$ \\
\hline \multicolumn{10}{|c|}{ Coconut Fronds } \\
\hline Kabelle & $3 f$ & 8 & $2.2 \times 10^{0}$ & $3.9 \times 10^{0}$ & $3.0 \times 10^{0}$ & $3.0 \times 10^{0}$ & $4.9 \times 10^{-1}$ & $1.1 \times 10^{0}$ & $1.7 \times 10^{-1}$ \\
\hline
\end{tabular}

NOTE: Specific activity is decay corrected to 1994 .

$N^{a}$ stands for number of individual samples. 


\section{Appendix B}

Radionuclide concentration summary in animal samples collected during the 1978 NMIRS and from 1986 through 1989 in the northern islands of Rongelap Atoll. 
Table B-1. The ${ }^{137} \mathrm{Cs}$ radionuclide concentration summary for animals (food source) collected from the northern islands of Rongelap Atoll during the 1978 NMIRS together with our recent trips in 1986 through 1989.

\begin{tabular}{|c|c|c|c|c|c|c|c|c|c|}
\hline \multirow{2}{*}{$\begin{array}{l}\text { Island } \\
\text { name }\end{array}$} & \multirow{2}{*}{$\begin{array}{l}\text { Island } \\
\text { code }\end{array}$} & \multirow[b]{2}{*}{$\mathrm{N}^{2}$} & \multicolumn{5}{|c|}{$\mathrm{pCi} \mathrm{g}^{-1}$ wet wt } & \multirow{2}{*}{$\begin{array}{l}\text { Mean } \\
\text { of logs }\end{array}$} & \multirow{2}{*}{$\begin{array}{c}\text { SD } \\
\text { of logs }\end{array}$} \\
\hline & & & Minimum & Maximum & Median & Mean & SD & & \\
\hline \multicolumn{10}{|c|}{ Coconut Crab Muscle } \\
\hline Naen & $01 \mathrm{f}$ & 1 & $1.4 \times 10^{1}$ & $1.4 \times 10^{1}$ & $1.4 \times 10^{1}$ & $1.4 \times 10^{1}$ & $0.0 \times 10^{0}$ & $2.7 \times 10^{0}$ & $0.0 \times 10^{0}$ \\
\hline Yugui & $05 f$ & 1 & $2.0 \times 10^{1}$ & $2.0 \times 10^{1}$ & $2.0 \times 10^{1}$ & $2.0 \times 10^{1}$ & $0.0 \times 10^{0}$ & $3.0 \times 10^{0}$ & $0.0 \times 10^{0}$ \\
\hline Lomiulal & $07 f$ & 2 & $7.9 \times 10^{0}$ & $1.3 \times 10^{1}$ & $1.0 \times 10^{1}$ & $1.0 \times 10^{1}$ & $3.4 \times 10^{0}$ & $2.3 \times 10^{0}$ & $3.3 \times 10^{-1}$ \\
\hline Kabelle & $13 \mathrm{f}$ & 10 & $3.0 \times 10^{0}$ & $9.8 \times 10^{0}$ & $4.0 \times 10^{0}$ & $5.2 \times 10^{0}$ & $2.5 \times 10^{0}$ & $1.5 \times 10^{0}$ & $4.6 \times 10^{-1}$ \\
\hline Mellu & $23 f$ & 3 & $2.7 \times 10^{0}$ & $5.5 \times 10^{0}$ & $3.5 \times 10^{0}$ & $3.9 \times 10^{0}$ & $1.4 \times 10^{0}$ & $1.3 \times 10^{0}$ & $3.6 \times 10^{-1}$ \\
\hline \multicolumn{10}{|c|}{ Coconut Crab Hepatopancreas } \\
\hline Naen & $01 f$ & 1 & $1.4 \times 10^{1}$ & $1.4 \times 10^{1}$ & $1.4 \times 10^{1}$ & $1.4 \times 10^{1}$ & $0.0 \times 10^{0}$ & $2.7 \times 10^{0}$ & $0.0 \times 10^{0}$ \\
\hline Yugui & $05 \mathrm{f}$ & 1 & $1.7 \times 10^{1}$ & $1.7 \times 10^{1}$ & $1.7 \times 10^{1}$ & $1.7 \times 10^{1}$ & $0.0 \times 10^{0}$ & $2.9 \times 10^{0}$ & $0.0 \times 10^{0}$ \\
\hline Lomiulal & $07 f$ & 1 & $7.0 \times 10^{0}$ & $7.0 \times 10^{0}$ & $7.0 \times 10^{0}$ & $7.0 \times 10^{0}$ & $0.0 \times 10^{0}$ & $1.9 \times 10^{0}$ & $0.0 \times 10^{0}$ \\
\hline Kabelle & $13 f$ & 9 & $1.9 \times 10^{0}$ & $1.6 \times 10^{1}$ & $5.7 \times 10^{0}$ & $7.6 \times 10^{0}$ & $4.7 \times 10^{0}$ & $1.8 \times 10^{0}$ & $6.8 \times 10^{-1}$ \\
\hline Mellu & $23 \mathrm{f}$ & 2 & $1.9 \times 10^{0}$ & $4.5 \times 10^{0}$ & $3.2 \times 10^{0}$ & $3.2 \times 10^{0}$ & $1.8 \times 10^{0}$ & $1.1 \times 10^{0}$ & $5.9 \times 10^{-1}$ \\
\hline
\end{tabular}

NOTE: Specific activity is decay corrected to 1994.

$\mathrm{N}^{\mathrm{a}}$ stands for number of individual samples. 


\section{Appendix C}

Radionuclide concentration summary of all soil profile samples collected during the 1978 NMIRS and from 1986 through 1989 in the northern islands of Rongelap Atoll. 
Table C-1. Cesium-137 radionuclide concentration summary for all soil profiles taken from the 1978 NMIRS together with our most recent trip in 1989 on Naen Island (F1).

\begin{tabular}{|c|c|c|c|c|c|c|c|c|}
\hline \multirow{2}{*}{$\begin{array}{l}\text { Soil } \\
\text { depth } \\
\text { (cm) }\end{array}$} & \multirow[b]{2}{*}{$\mathrm{Na}$} & \multicolumn{5}{|c|}{ pCi g ${ }^{-1}$ dry wt } & \multirow{2}{*}{$\begin{array}{l}\text { Mean } \\
\text { of logs } \\
\end{array}$} & \multirow{2}{*}{$\begin{array}{c}\mathrm{SD} \\
\text { of logs } \\
\end{array}$} \\
\hline & & Minimum & Maximum & Median & Mean & $\mathrm{SD}$ & & \\
\hline $0-05$ & 16 & $1.1 \times 10^{1}$ & $4.0 \times 10^{2}$ & $8.3 \times 10^{1}$ & $1.2 \times 10^{2}$ & $1.1 \times 10^{2}$ & $4.4 \times 10^{0}$ & $9.3 \times 10^{-1}$ \\
\hline $05-10$ & 17 & $1.3 \times 10^{1}$ & $1.0 \times 10^{2}$ & $4.4 \times 10^{1}$ & $4.6 \times 10^{1}$ & $2.4 \times 10^{1}$ & $3.7 \times 10^{0}$ & $6.0 \times 10^{-1}$ \\
\hline $10-15$ & 17 & $4.0 \times 10^{0}$ & $7.3 \times 10^{1}$ & $2.1 \times 10^{1}$ & $2.3 \times 10^{1}$ & $1.6 \times 10^{1}$ & $3.0 \times 10^{0}$ & $6.7 \times 10^{-1}$ \\
\hline $15-25$ & 17 & $1.5 \times 10^{0}$ & $1.5 \times 10^{1}$ & $7.7 \times 10^{0}$ & $7.1 \times 10^{0}$ & $3.5 \times 10^{0}$ & $1.8 \times 10^{0}$ & $5.8 \times 10^{-1}$ \\
\hline $25-40$ & 16 & $2.9 \times 10^{-1}$ & $5.6 \times 10^{0}$ & $1.5 \times 10^{0}$ & $1.9 \times 10^{0}$ & $1.5 \times 10^{0}$ & $3.8 \times 10^{-1}$ & $7.8 \times 10^{-1}$ \\
\hline $40-60$ & 7 & $1.9 \times 10^{-1}$ & $4.9 \times 10^{0}$ & $1.5 \times 10^{0}$ & $1.7 \times 10^{0}$ & $1.6 \times 10^{0}$ & $2.8 \times 10^{-2}$ & $1.2 \times 10^{0}$ \\
\hline $0-05$ & 16 & $1.1 \times 10^{1}$ & $4.0 \times 10^{2}$ & $8.3 \times 10^{1}$ & $1.2 \times 10^{2}$ & $1.1 \times 10^{2}$ & $4.4 \times 10^{0}$ & $9.3 \times 10^{-1}$ \\
\hline $0-10$ & 16 & $1.5 \times 10^{1}$ & $2.2 \times 10^{2}$ & $6.9 \times 10^{1}$ & $8.4 \times 10^{1}$ & $5.8 \times 10^{1}$ & $4.2 \times 10^{0}$ & $7.5 \times 10^{-1}$ \\
\hline $0-15$ & 16 & $1.8 \times 10^{1}$ & $1.5 \times 10^{2}$ & $5.3 \times 10^{1}$ & $6.4 \times 10^{1}$ & $3.9 \times 10^{1}$ & $4.0 \times 10^{0}$ & $6.4 \times 10^{-1}$ \\
\hline $0-25$ & 16 & $1.4 \times 10^{1}$ & $9.5 \times 10^{1}$ & $3.6 \times 10^{1}$ & $4.1 \times 10^{1}$ & $2.3 \times 10^{1}$ & $3.6 \times 10^{0}$ & $5.7 \times 10^{-1}$ \\
\hline $0-40$ & 15 & $9.1 \times 10^{0}$ & $6.0 \times 10^{1}$ & $2.4 \times 10^{1}$ & $2.6 \times 10^{1}$ & $1.5 \times 10^{1}$ & $3.1 \times 10^{0}$ & $5.7 \times 10^{-1}$ \\
\hline $0-60$ & 6 & $6.1 \times 10^{0}$ & $2.3 \times 10^{1}$ & $1.3 \times 10^{1}$ & $1.4 \times 10^{1}$ & $7.2 \times 10^{0}$ & $2.6 \times 10^{0}$ & $5.4 \times 10^{-1}$ \\
\hline
\end{tabular}

NOTE: Specific activity is decay corrected to 1994.

$\mathrm{N}^{\mathrm{a}}$ stands for number of individual samples. 
Table C-2. Cesium-137 radionuclide concentration summary for all soil profiles taken from the 1978 NMIRS together with our most recent trip in 1989 on Yugui Island (F5).

\begin{tabular}{rrrlrcccc}
\hline $\begin{array}{c}\text { Soil } \\
\text { depth } \\
\text { (cm) }\end{array}$ & Na & Minimum & \multicolumn{7}{c}{ Maximum } & Median & Mean & SD & $\begin{array}{c}\text { Mean } \\
\text { of logs }\end{array}$ & $\begin{array}{c}\text { SD } \\
\text { of logs }\end{array}$ \\
\hline $0-05$ & 11 & $4.3 \times 10^{1}$ & $3.0 \times 10^{2}$ & $1.7 \times 10^{2}$ & $1.7 \times 10^{2}$ & $9.0 \times 10^{1}$ & $5.0 \times 10^{0}$ & $6.7 \times 10^{-1}$ \\
$05-10$ & 11 & $7.4 \times 10^{0}$ & $2.7 \times 10^{2}$ & $7.3 \times 10^{1}$ & $8.6 \times 10^{1}$ & $7.1 \times 10^{1}$ & $4.1 \times 10^{0}$ & $9.4 \times 10^{-1}$ \\
$10-15$ & 11 & $3.6 \times 10^{0}$ & $6.0 \times 10^{1}$ & $3.2 \times 10^{1}$ & $2.8 \times 10^{1}$ & $1.6 \times 10^{1}$ & $3.1 \times 10^{0}$ & $8.1 \times 10^{-1}$ \\
$15-25$ & 11 & $2.7 \times 10^{0}$ & $3.0 \times 10^{1}$ & $8.8 \times 10^{0}$ & $9.7 \times 10^{0}$ & $7.9 \times 10^{0}$ & $2.0 \times 10^{0}$ & $7.3 \times 10^{-1}$ \\
$25-40$ & 11 & $1.7 \times 10^{0}$ & $6.4 \times 10^{0}$ & $2.8 \times 10^{0}$ & $3.2 \times 10^{0}$ & $1.4 \times 10^{0}$ & $1.1 \times 10^{0}$ & $4.2 \times 10^{-1}$ \\
$40-60$ & 1 & $5.3 \times 10^{-1}$ & $5.3 \times 10^{-1}$ & $5.3 \times 10^{-1}$ & $5.3 \times 10^{-1}$ & $0.0 \times 10^{0}$ & $-6.5 \times 10^{-1}$ & $0.0 \times 10^{0}$ \\
& & & & & & & & \\
& & & & & & & \\
$0-05$ & 11 & $4.3 \times 10^{1}$ & $3.0 \times 10^{2}$ & $1.7 \times 10^{2}$ & $1.7 \times 10^{2}$ & $9.0 \times 10^{1}$ & $5.0 \times 10^{0}$ & $6.7 \times 10^{-1}$ \\
$0-10$ & 11 & $2.5 \times 10^{1}$ & $2.1 \times 10^{2}$ & $1.4 \times 10^{2}$ & $1.3 \times 10^{2}$ & $5.4 \times 10^{1}$ & $4.7 \times 10^{0}$ & $6.2 \times 10^{-1}$ \\
$0-15$ & 11 & $1.8 \times 10^{1}$ & $1.5 \times 10^{2}$ & $1.0 \times 10^{2}$ & $9.5 \times 10^{1}$ & $3.9 \times 10^{1}$ & $4.4 \times 10^{0}$ & $6.1 \times 10^{-1}$ \\
$0-25$ & 11 & $1.3 \times 10^{1}$ & $9.4 \times 10^{1}$ & $6.5 \times 10^{1}$ & $6.1 \times 10^{1}$ & $2.5 \times 10^{1}$ & $4.0 \times 10^{0}$ & $5.9 \times 10^{-1}$ \\
$0-40$ & 11 & $1.0 \times 10^{1}$ & $6.0 \times 10^{1}$ & $4.2 \times 10^{1}$ & $3.9 \times 10^{1}$ & $1.5 \times 10^{1}$ & $3.6 \times 10^{0}$ & $5.3 \times 10^{-1}$ \\
$0-60$ & 1 & $1.4 \times 10^{1}$ & $1.4 \times 10^{1}$ & $1.4 \times 10^{1}$ & $1.4 \times 10^{1}$ & $0.0 \times 10^{0}$ & $2.7 \times 10^{0}$ & $0.0 \times 10^{0}$ \\
\hline
\end{tabular}

NOTE: Specific activity is decay corrected to 1994.

$\mathrm{N}^{\mathrm{a}}$ stands for number of individual samples. 
Table C-3. Cesium-137 radionuclide concentration summary for all soil profiles taken from the 1978 NMIRS together with our most recent trip in 1989 on Lomiulal Island (F7).

\begin{tabular}{|c|c|c|c|c|c|c|c|c|}
\hline \multirow{2}{*}{$\begin{array}{l}\text { Soil } \\
\text { depth } \\
\text { (cm) }\end{array}$} & \multirow[b]{2}{*}{$\mathrm{Na}^{\mathrm{a}}$} & \multicolumn{5}{|c|}{ pCi g-1 dry wt } & \multirow{2}{*}{$\begin{array}{l}\text { Mean } \\
\text { of logs }\end{array}$} & \multirow{2}{*}{$\begin{array}{c}\text { SD } \\
\text { of logs } \\
\end{array}$} \\
\hline & & Minimum & Maximum & Median & Mean & $\mathrm{SD}$ & & \\
\hline $0-05$ & 14 & $3.2 \times 10^{0}$ & $2.3 \times 10^{2}$ & $5.3 \times 10^{1}$ & $8.1 \times 10^{1}$ & $6.7 \times 10^{1}$ & $4.0 \times 10^{0}$ & $1.1 \times 10^{0}$ \\
\hline $05-10$ & 14 & $1.2 \times 10^{1}$ & $5.7 \times 10^{1}$ & $3.7 \times 10^{1}$ & $3.5 \times 10^{1}$ & $1.4 \times 10^{1}$ & $3.5 \times 10^{0}$ & $4.8 \times 10^{-1}$ \\
\hline $10-15$ & 14 & $3.9 \times 10^{0}$ & $4.6 \times 10^{1}$ & $1.5 \times 10^{1}$ & $1.8 \times 10^{1}$ & $1.1 \times 10^{1}$ & $2.7 \times 10^{0}$ & $6.1 \times 10^{-1}$ \\
\hline $15-25$ & 14 & $1.8 \times 10^{0}$ & $2.4 \times 10^{1}$ & $6.3 \times 10^{0}$ & $9.4 \times 10^{0}$ & $7.6 \times 10^{0}$ & $1.9 \times 10^{0}$ & $8.1 \times 10^{-1}$ \\
\hline $25-40$ & 14 & $6.1 \times 10^{-1}$ & $1.0 \times 10^{1}$ & $1.5 \times 10^{0}$ & $3.2 \times 10^{0}$ & $3.5 \times 10^{0}$ & $6.6 \times 10^{-1}$ & $1.0 \times 10^{0}$ \\
\hline $40-60$ & 3 & $9.0 \times 10^{-2}$ & $2.8 \times 10^{0}$ & $3.9 \times 10^{-1}$ & $1.1 \times 10^{0}$ & $1.5 \times 10^{0}$ & $-7.8 \times 10^{-1}$ & $1.7 \times 10^{0}$ \\
\hline $0-05$ & 14 & $3.2 \times 10^{0}$ & $2.3 \times 10^{2}$ & $5.3 \times 10^{1}$ & $8.1 \times 10^{1}$ & $6.7 \times 10^{1}$ & $4.0 \times 10^{0}$ & $1.1 \times 10^{0}$ \\
\hline $0-10$ & 14 & $1.8 \times 10^{1}$ & $1.3 \times 10^{2}$ & $4.7 \times 10^{1}$ & $5.8 \times 10^{1}$ & $3.6 \times 10^{1}$ & $3.9 \times 10^{0}$ & $6.4 \times 10^{-1}$ \\
\hline $0-15$ & 14 & $1.7 \times 10^{1}$ & $9.0 \times 10^{1}$ & $3.9 \times 10^{1}$ & $4.4 \times 10^{1}$ & $2.4 \times 10^{1}$ & $3.7 \times 10^{0}$ & $5.5 \times 10^{-1}$ \\
\hline $0-25$ & 14 & $1.3 \times 10^{1}$ & $5.6 \times 10^{1}$ & $3.0 \times 10^{1}$ & $3.0 \times 10^{1}$ & $1.4 \times 10^{1}$ & $3.3 \times 10^{0}$ & $4.7 \times 10^{-1}$ \\
\hline $0-40$ & 14 & $8.5 \times 10^{0}$ & $3.6 \times 10^{1}$ & $2.1 \times 10^{1}$ & $2.0 \times 10^{1}$ & $8.6 \times 10^{0}$ & $2.9 \times 10^{0}$ & $4.5 \times 10^{-1}$ \\
\hline $0-60$ & 3 & $6.4 \times 10^{0}$ & $1.1 \times 10^{1}$ & $9.5 \times 10^{0}$ & $8.9 \times 10^{0}$ & $2.3 \times 10^{0}$ & $2.2 \times 10^{0}$ & $2.8 \times 10^{-1}$ \\
\hline
\end{tabular}

NOTE: Specific activity is decay corrected to 1994.

$\mathrm{N}^{\mathrm{a}}$ stands for number of individual samples. 
Table C-4. Cesium-137 radionuclide concentration summary for all soil profiles taken from the 1978 NMIRS together with our most recent trips in 1988 through 1989 on Kabelle Island (F13).

\begin{tabular}{|c|c|c|c|c|c|c|c|c|}
\hline \multirow{2}{*}{$\begin{array}{c}\text { Soil } \\
\text { depth } \\
(\mathrm{cm})\end{array}$} & \multirow[b]{2}{*}{$\mathrm{Na}^{\mathrm{a}}$} & \multicolumn{5}{|c|}{ pCi g $^{-1}$ dry wt } & \multirow{2}{*}{$\begin{array}{r}\text { Mean } \\
\text { of logs }\end{array}$} & \multirow{2}{*}{$\begin{array}{c}\text { SD } \\
\text { of logs } \\
\end{array}$} \\
\hline & & Minimum & Maximum & Median & Mean & $\overline{\mathrm{SD}}$ & & \\
\hline $0-05$ & 34 & $1.0 \times 10^{-1}$ & $1.2 \times 10^{2}$ & $2.8 \times 10^{2}$ & $3.4 \times 10^{1}$ & $2.8 \times 10^{1}$ & $3.0 \times 10^{0}$ & $1.4 \times 10^{0}$ \\
\hline $05-10$ & 16 & $5.8 \times 10^{-2}$ & $3.9 \times 10^{1}$ & $1.4 \times 10^{1}$ & $1.5 \times 10^{1}$ & $1.1 \times 10^{1}$ & $2.2 \times 10^{0}$ & $1.6 \times 10^{0}$ \\
\hline $10-15$ & 17 & $2.6 \times 10^{-1}$ & $2.5 \times 10^{1}$ & $7.6 \times 10^{0}$ & $9.4 \times 10^{0}$ & $7.0 \times 10^{0}$ & $1.9 \times 10^{0}$ & $1.1 \times 10^{0}$ \\
\hline $15-25$ & 17 & $7.5 \times 10^{-1}$ & $2.2 \times 10^{1}$ & $3.2 \times 10^{0}$ & $6.2 \times 10^{0}$ & $6.5 \times 10^{0}$ & $1.4 \times 10^{0}$ & $1.0 \times 10^{0}$ \\
\hline $25-40$ & 17 & $1.3 \times 10^{-1}$ & $1.1 \times 10^{1}$ & $1.4 \times 10^{0}$ & $2.1 \times 10^{0}$ & $2.7 \times 10^{0}$ & $9.7 \times 10^{-2}$ & $1.2 \times 10^{0}$ \\
\hline $40-60$ & 6 & $1.2 \times 10^{-1}$ & $1.1 \times 10^{0}$ & $4.2 \times 10^{-1}$ & $4.8 \times 10^{-1}$ & $3.5 \times 10^{-1}$ & $-9.9 \times 10^{-1}$ & $8.2 \times 10^{-1}$ \\
\hline $0-05$ & 34 & $1.0 \times 10^{-1}$ & $1.2 \times 10^{2}$ & $2.8 \times 10^{1}$ & $3.4 \times 10^{1}$ & $2.8 \times 10^{1}$ & $3.0 \times 10^{0}$ & $1.4 \times 10^{0}$ \\
\hline $0-10$ & 16 & $7.9 \times 10^{-2}$ & $6.3 \times 10^{1}$ & $1.9 \times 10^{1}$ & $2.2 \times 10^{1}$ & $1.7 \times 10^{1}$ & $2.5 \times 10^{0}$ & $1.6 \times 10^{0}$ \\
\hline $0-15$ & 16 & $1.4 \times 10^{-1}$ & $4.3 \times 10^{1}$ & $1.8 \times 10^{1}$ & $1.8 \times 10^{1}$ & $1.2 \times 10^{1}$ & $2.4 \times 10^{0}$ & $1.4 \times 10^{0}$ \\
\hline $0-25$ & 16 & $3.9 \times 10^{-1}$ & $2.6 \times 10^{1}$ & $1.4 \times 10^{1}$ & $1.3 \times 10^{1}$ & $7.8 \times 10^{0}$ & $2.2 \times 10^{0}$ & $1.1 \times 10^{0}$ \\
\hline $0-40$ & 16 & $2.2 \times 10^{0}$ & $1.8 \times 10^{1}$ & $9.2 \times 10^{0}$ & $8.8 \times 10^{0}$ & $5.2 \times 10^{0}$ & $2.0 \times 10^{0}$ & $7.2 \times 10^{-1}$ \\
\hline $0-60$ & 6 & $1.6 \times 10^{0}$ & $1.2 \times 10^{1}$ & $5.6 \times 10^{0}$ & $5.9 \times 10^{0}$ & $4.2 \times 10^{0}$ & $1.5 \times 10^{0}$ & $8.4 \times 10^{-1}$ \\
\hline
\end{tabular}

NOTE: Specific activity is decay corrected to 1994.

$\mathrm{N}^{\mathrm{a}}$ stands for number of individual samples. 
Table C-5. Cesium-137 radionuclide concentration summary for all soil profiles taken from the 1978 NMIRS together with our most recent trip in 1989 on Mellu Island (F23).

\begin{tabular}{|c|c|c|c|c|c|c|c|c|}
\hline \multirow{2}{*}{$\begin{array}{l}\text { Soil } \\
\text { depth } \\
\text { (cm) }\end{array}$} & \multirow[b]{2}{*}{$\mathrm{Na}^{\mathrm{a}}$} & \multicolumn{5}{|c|}{$\mathrm{pCig}^{-1}$ dry wt } & \multirow{2}{*}{$\begin{array}{l}\text { Mean } \\
\text { of logs } \\
\end{array}$} & \multirow{2}{*}{$\begin{array}{c}\text { SD } \\
\text { of logs }\end{array}$} \\
\hline & & Minimum & Maximum & Median & Mean & $\mathrm{SD}$ & & \\
\hline $0-05$ & 15 & $1.1 \times 10^{1}$ & $1.0 \times 10^{2}$ & $3.2 \times 10^{1}$ & $3.8 \times 10^{1}$ & $2.8 \times 10^{1}$ & $3.4 \times 10^{0}$ & $7.3 \times 10^{-1}$ \\
\hline $05-10$ & 15 & $1.2 \times 10^{1}$ & $5.7 \times 10^{1}$ & $1.9 \times 10^{1}$ & $2.9 \times 10^{1}$ & $1.7 \times 10^{1}$ & $3.2 \times 10^{0}$ & $5.7 \times 10^{-1}$ \\
\hline $10-15$ & 15 & $9.2 \times 10^{0}$ & $4.7 \times 10^{1}$ & $1.3 \times 10^{1}$ & $1.7 \times 10^{1}$ & $1.0 \times 10^{1}$ & $2.7 \times 10^{0}$ & $4.7 \times 10^{-1}$ \\
\hline $15-25$ & 15 & $3.2 \times 10^{0}$ & $3.5 \times 10^{1}$ & $9.9 \times 10^{0}$ & $1.1 \times 10^{1}$ & $8.7 \times 10^{0}$ & $2.2 \times 10^{0}$ & $7.2 \times 10^{-1}$ \\
\hline $25-40$ & 14 & $9.7 \times 10^{-1}$ & $1.2 \times 10^{1}$ & $4.0 \times 10^{0}$ & $5.0 \times 10^{0}$ & $3.7 \times 10^{0}$ & $1.3 \times 10^{0}$ & $7.8 \times 10^{-1}$ \\
\hline $40-60$ & 1 & $2.0 \times 10^{0}$ & $2.0 \times 10^{0}$ & $2.0 \times 10^{0}$ & $2.0 \times 10^{0}$ & $0.0 \times 10^{0}$ & $6.8 \times 10^{-1}$ & $0.0 \times 10^{0}$ \\
\hline $0-05$ & 15 & $1.1 \times 10^{1}$ & $1.0 \times 10^{2}$ & $3.2 \times 10^{1}$ & $3.8 \times 10^{1}$ & $2.8 \times 10^{1}$ & $3.4 \times 10^{0}$ & $7.3 \times 10^{-1}$ \\
\hline $0-10$ & 15 & $1.1 \times 10^{1}$ & $7.9 \times 10^{1}$ & $2.7 \times 10^{1}$ & $3.4 \times 10^{1}$ & $2.1 \times 10^{1}$ & $3.3 \times 10^{0}$ & $6.1 \times 10^{-1}$ \\
\hline $0-15$ & 15 & $1.2 \times 10^{1}$ & $6.1 \times 10^{1}$ & $2.2 \times 10^{1}$ & $2.8 \times 10^{1}$ & $1.6 \times 10^{1}$ & $3.2 \times 10^{0}$ & $5.4 \times 10^{-1}$ \\
\hline $0-25$ & 15 & $1.0 \times 10^{1}$ & $5.1 \times 10^{1}$ & $1.6 \times 10^{1}$ & $2.1 \times 10^{1}$ & $1.2 \times 10^{1}$ & $2.9 \times 10^{0}$ & $5.0 \times 10^{-1}$ \\
\hline $0-40$ & 14 & $8.0 \times 10^{0}$ & $3.3 \times 10^{1}$ & $1.3 \times 10^{1}$ & $1.6 \times 10^{1}$ & $7.9 \times 10^{0}$ & $2.7 \times 10^{0}$ & $4.6 \times 10^{-1}$ \\
\hline $0-60$ & 1 & $2.1 \times 10^{1}$ & $2.1 \times 10^{1}$ & $2.1 \times 10^{1}$ & $2.1 \times 10^{1}$ & $0.0 \times 10^{0}$ & $3.0 \times 10^{0}$ & $0.0 \times 10^{0}$ \\
\hline
\end{tabular}

NOTE: Specific activity is decay corrected to 1994.

$\mathrm{N}^{\mathrm{a}}$ stands for number of individual samples. 
Table C-6. Strontium-90 radionuclide concentration summary for all soil profiles taken during the 1978 NMIRS on Naen Island (F1).

\begin{tabular}{rcccccccc}
\hline $\begin{array}{c}\text { Soil } \\
\text { depth } \\
\text { (cm) }\end{array}$ & Na & Minimum & Maximum & Median & Mean & SD & $\begin{array}{c}\text { Mean } \\
\text { of logs }\end{array}$ & $\begin{array}{c}\text { SD } \\
\text { of logs }\end{array}$ \\
\hline $0-05$ & 7 & $4.7 \times 10^{1}$ & $1.7 \times 10^{2}$ & $1.1 \times 10^{2}$ & $1.1 \times 10^{2}$ & $4.0 \times 10^{1}$ & $4.6 \times 10^{0}$ & $4.2 \times 10^{-1}$ \\
$05-10$ & 7 & $1.1 \times 10^{1}$ & $1.3 \times 10^{2}$ & $7.7 \times 10^{1}$ & $7.9 \times 10^{1}$ & $3.9 \times 10^{1}$ & $4.2 \times 10^{0}$ & $8.3 \times 10^{-1}$ \\
$10-15$ & 7 & $6.6 \times 10^{0}$ & $9.5 \times 10^{1}$ & $4.1 \times 10^{1}$ & $5.1 \times 10^{1}$ & $3.5 \times 10^{1}$ & $3.6 \times 10^{0}$ & $9.6 \times 10^{-1}$ \\
$15-25$ & 7 & $2.0 \times 10^{0}$ & $4.8 \times 10^{1}$ & $1.0 \times 10^{1}$ & $1.5 \times 10^{1}$ & $1.6 \times 10^{1}$ & $2.2 \times 10^{0}$ & $1.1 \times 10^{0}$ \\
$25-40$ & 7 & $1.6 \times 10^{0}$ & $3.6 \times 10^{1}$ & $6.9 \times 10^{0}$ & $9.7 \times 10^{0}$ & $1.2 \times 10^{1}$ & $1.8 \times 10^{0}$ & $9.5 \times 10^{-1}$ \\
$40-60$ & 7 & $1.2 \times 10^{0}$ & $6.6 \times 10^{0}$ & $3.6 \times 10^{0}$ & $3.7 \times 10^{0}$ & $1.9 \times 10^{0}$ & $1.2 \times 10^{0}$ & $5.8 \times 10^{-1}$ \\
& & & & & & & & \\
$0-05$ & 7 & $4.7 \times 10^{1}$ & $1.7 \times 10^{2}$ & $1.1 \times 10^{2}$ & $1.1 \times 10^{2}$ & $4.0 \times 10^{1}$ & $4.6 \times 10^{0}$ & $4.2 \times 10^{-1}$ \\
$0-10$ & 7 & $4.6 \times 10^{1}$ & $1.4 \times 10^{2}$ & $8.7 \times 10^{1}$ & $9.3 \times 10^{1}$ & $3.5 \times 10^{1}$ & $4.5 \times 10^{0}$ & $4.1 \times 10^{-1}$ \\
$0-15$ & 7 & $3.3 \times 10^{1}$ & $1.2 \times 10^{2}$ & $7.0 \times 10^{1}$ & $7.9 \times 10^{1}$ & $3.0 \times 10^{1}$ & $4.3 \times 10^{0}$ & $4.3 \times 10^{-1}$ \\
$0-25$ & 7 & $2.1 \times 10^{1}$ & $8.2 \times 10^{1}$ & $4.7 \times 10^{1}$ & $5.3 \times 10^{1}$ & $2.0 \times 10^{1}$ & $3.9 \times 10^{0}$ & $4.5 \times 10^{-1}$ \\
$0-40$ & 7 & $1.6 \times 10^{1}$ & $6.5 \times 10^{1}$ & $3.2 \times 10^{1}$ & $3.7 \times 10^{1}$ & $1.6 \times 10^{1}$ & $3.5 \times 10^{0}$ & $4.4 \times 10^{-1}$ \\
$0-60$ & 7 & $1.1 \times 10^{1}$ & $4.5 \times 10^{1}$ & $2.3 \times 10^{1}$ & $2.6 \times 10^{1}$ & $1.1 \times 10^{1}$ & $3.2 \times 10^{0}$ & $4.3 \times 10^{-1}$ \\
\hline
\end{tabular}

NOTE: Specific activity is decay corrected to 1994.

$\mathrm{N}^{\mathrm{a}}$ stands for number of individual samples. 
Table C-7. Strontium-90 radionuclide concentration summary for all soil profiles taken during the 1978 NMIRS on Yugui Island (F5).

\begin{tabular}{|c|c|c|c|c|c|c|c|c|}
\hline \multirow{2}{*}{$\begin{array}{r}\text { Soil } \\
\text { depth } \\
(\mathrm{cm})\end{array}$} & \multirow[b]{2}{*}{$\mathrm{Na}^{\mathrm{a}}$} & \multicolumn{5}{|c|}{ pCi g ${ }^{-1}$ dry wt } & \multirow{2}{*}{$\begin{array}{l}\text { Mean } \\
\text { of logs }\end{array}$} & \multirow{2}{*}{$\begin{array}{c}\mathrm{SD} \\
\text { of logs } \\
\end{array}$} \\
\hline & & Minimum & Maximum & Median & Mean & $\mathrm{SD}$ & & \\
\hline $0-05$ & 1 & $3.2 \times 10^{1}$ & $3.2 \times 10^{1}$ & $3.2 \times 10^{1}$ & $3.2 \times 10^{1}$ & $0.0 \times 10^{0}$ & $3.5 \times 10^{0}$ & $0.0 \times 10^{0}$ \\
\hline $05-10$ & 1 & $2.0 \times 10^{1}$ & $2.0 \times 10^{1}$ & $2.0 \times 10^{1}$ & $2.0 \times 10^{1}$ & $0.0 \times 10^{0}$ & $3.0 \times 10^{0}$ & $0.0 \times 10^{0}$ \\
\hline $10-15$ & 1 & $1.1 \times 10^{1}$ & $1.1 \times 10^{1}$ & $1.1 \times 10^{1}$ & $1.1 \times 10^{1}$ & $0.0 \times 10^{0}$ & $2.4 \times 10^{0}$ & $0.0 \times 10^{0}$ \\
\hline $15-25$ & 1 & $3.6 \times 10^{0}$ & $3.6 \times 10^{0}$ & $3.6 \times 10^{0}$ & $3.6 \times 10^{0}$ & $0.0 \times 10^{0}$ & $1.3 \times 10^{0}$ & $0.0 \times 10^{0}$ \\
\hline $25-40$ & 1 & $2.7 \times 10^{0}$ & $2.7 \times 10^{0}$ & $2.7 \times 10^{0}$ & $2.7 \times 10^{0}$ & $0.0 \times 10^{0}$ & $1.0 \times 10^{0}$ & $0.0 \times 10^{0}$ \\
\hline $40-60$ & 1 & $2.1 \times 10^{-1}$ & $2.1 \times 10^{-1}$ & $2.1 \times 10^{-1}$ & $2.1 \times 10^{-1}$ & $0.0 \times 10^{0}$ & $-1.6 \times 10^{0}$ & $0.0 \times 10^{0}$ \\
\hline $0-05$ & 1 & $3.2 \times 10^{1}$ & $3.2 \times 10^{1}$ & $3.2 \times 10^{1}$ & $3.2 \times 10^{1}$ & $0.0 \times 10^{0}$ & $3.5 \times 10^{0}$ & $0.0 \times 10^{0}$ \\
\hline $0-10$ & 1 & $2.6 \times 10^{1}$ & $2.6 \times 10^{1}$ & $2.6 \times 10^{1}$ & $2.6 \times 10^{1}$ & $0.0 \times 10^{0}$ & $3.3 \times 10^{0}$ & $0.0 \times 10^{0}$ \\
\hline $0-15$ & 1 & $2.1 \times 10^{1}$ & $2.1 \times 10^{1}$ & $2.1 \times 10^{1}$ & $2.1 \times 10^{1}$ & $0.0 \times 10^{0}$ & $3.1 \times 10^{0}$ & $0.0 \times 10^{0}$ \\
\hline $0-25$ & 1 & $1.4 \times 10^{1}$ & $1.4 \times 10^{1}$ & $1.4 \times 10^{1}$ & $1.4 \times 10^{1}$ & $0.0 \times 10^{0}$ & $2.7 \times 10^{0}$ & $0.0 \times 10^{0}$ \\
\hline $0-40$ & 1 & $9.9 \times 10^{0}$ & $9.9 \times 10^{0}$ & $9.9 \times 10^{0}$ & $9.9 \times 10^{0}$ & $0.0 \times 10^{0}$ & $2.3 \times 10^{0}$ & $0.0 \times 10^{0}$ \\
\hline $0-60$ & 1 & $6.7 \times 10^{0}$ & $6.7 \times 10^{0}$ & $6.7 \times 10^{0}$ & $6.7 \times 10^{0}$ & $0.0 \times 10^{0}$ & $1.9 \times 10^{0}$ & $0.0 \times 10^{0}$ \\
\hline
\end{tabular}

NOTE: Specific activity is decay corrected to 1994 .

$\mathrm{N}^{\mathrm{a}}$ stands for number of individual samples. 
Table C-8. Strontium-90 radionuclide concentration summary for all soil profiles taken during the 1978 NMIRS on Lomiulal Island (F7).

\begin{tabular}{|c|c|c|c|c|c|c|c|c|}
\hline \multirow{2}{*}{$\begin{array}{c}\text { Soil } \\
\text { depth } \\
\text { (cm) }\end{array}$} & \multirow[b]{2}{*}{$\mathrm{Na}$} & \multicolumn{5}{|c|}{$\mathrm{pCi} \mathrm{g}^{-1}$ dry wt } & \multirow{2}{*}{$\begin{array}{r}\text { Mean } \\
\text { of } \operatorname{logs} \\
\end{array}$} & \multirow{2}{*}{$\begin{array}{c}\text { SD } \\
\text { of } \operatorname{logs} \\
\end{array}$} \\
\hline & & Minimum & Maximum & Median & Mean & $\overline{\mathrm{SD}}$ & & \\
\hline $0-05$ & 4 & $2.2 \times 10^{1}$ & $1.4 \times 10^{2}$ & $5.1 \times 10^{1}$ & $6.6 \times 10^{1}$ & $5.2 \times 10^{1}$ & $4.0 \times 10^{0}$ & $7.9 \times 10^{-1}$ \\
\hline $05-10$ & 4 & $2.0 \times 10^{1}$ & $6.7 \times 10^{1}$ & $4.9 \times 10^{1}$ & $4.6 \times 10^{1}$ & $2.1 \times 10^{1}$ & $3.7 \times 10^{0}$ & $5.4 \times 10^{-1}$ \\
\hline $10-15$ & 4 & $1.1 \times 10^{1}$ & $3.7 \times 10^{1}$ & $2.6 \times 10^{1}$ & $2.5 \times 10^{1}$ & $1.1 \times 10^{1}$ & $3.1 \times 10^{0}$ & $5.1 \times 10^{-1}$ \\
\hline $15-25$ & 4 & $6.8 \times 10^{0}$ & $3.2 \times 10^{1}$ & $1.2 \times 10^{1}$ & $1.6 \times 10^{1}$ & $1.1 \times 10^{1}$ & $2.6 \times 10^{0}$ & $6.5 \times 10^{-1}$ \\
\hline $25-40$ & 4 & $1.4 \times 10^{0}$ & $1.7 \times 10^{1}$ & $6.3 \times 10^{0}$ & $7.8 \times 10^{0}$ & $6.6 \times 10^{0}$ & $1.7 \times 10^{0}$ & $1.0 \times 10^{0}$ \\
\hline $40-60$ & 4 & $4.9 \times 10^{-1}$ & $8.1 \times 10^{0}$ & $2.6 \times 10^{0}$ & $3.4 \times 10^{0}$ & $3.3 \times 10^{0}$ & $8.1 \times 10^{-1}$ & $1.2 \times 10^{0}$ \\
\hline $0-05$ & 4 & $2.2 \times 10^{1}$ & $1.4 \times 10^{2}$ & $5.1 \times 10^{1}$ & $6.6 \times 10^{1}$ & $5.2 \times 10^{1}$ & $4.0 \times 10^{0}$ & $7.9 \times 10^{-1}$ \\
\hline $0-10$ & 4 & $3.0 \times 10^{1}$ & $1.0 \times 10^{2}$ & $4.5 \times 10^{1}$ & $5.6 \times 10^{1}$ & $3.3 \times 10^{1}$ & $3.9 \times 10^{0}$ & $5.2 \times 10^{-1}$ \\
\hline $0-15$ & 4 & $3.2 \times 10^{1}$ & $7.7 \times 10^{1}$ & $3.7 \times 10^{1}$ & $4.6 \times 10^{1}$ & $2.1 \times 10^{1}$ & $3.8 \times 10^{0}$ & $4.1 \times 10^{-1}$ \\
\hline $0-25$ & 4 & $2.5 \times 10^{1}$ & $4.9 \times 10^{1}$ & $3.1 \times 10^{1}$ & $3.4 \times 10^{1}$ & $1.1 \times 10^{1}$ & $3.5 \times 10^{0}$ & $2.9 \times 10^{-1}$ \\
\hline $0-40$ & 4 & $1.8 \times 10^{1}$ & $3.1 \times 10^{1}$ & $2.4 \times 10^{1}$ & $2.4 \times 10^{1}$ & $5.9 \times 10^{0}$ & $3.2 \times 10^{0}$ & $2.5 \times 10^{-1}$ \\
\hline $0-60$ & 4 & $1.3 \times 10^{1}$ & $2.1 \times 10^{1}$ & $1.7 \times 10^{1}$ & $1.7 \times 10^{1}$ & $4.1 \times 10^{0}$ & $2.8 \times 10^{0}$ & $2.5 \times 10^{-1}$ \\
\hline
\end{tabular}

NOTE: Specific activity is decay corrected to 1994.

$\mathrm{N}^{\mathrm{a}}$ stands for number of individual samples. 
Table C-9. Strontium-90 radionuclide concentration summary for all soil profiles taken during the 1978 NMIRS on Kabelle Island (F13).

\begin{tabular}{|c|c|c|c|c|c|c|c|c|}
\hline \multirow{2}{*}{$\begin{array}{l}\text { Soil } \\
\text { depth } \\
(\mathrm{cm})\end{array}$} & \multirow[b]{2}{*}{$\mathrm{Na}$} & \multicolumn{5}{|c|}{ pCi g $g^{-1}$ dry wt } & \multirow{2}{*}{$\begin{array}{l}\text { Mean } \\
\text { of logs }\end{array}$} & \multirow{2}{*}{$\begin{array}{c}\text { SD } \\
\text { of } \operatorname{logs}\end{array}$} \\
\hline & & Minimum & Maximum & Median & Mean & $\overline{\mathrm{SD}}$ & & \\
\hline $0-05$ & 5 & $6.4 \times 10^{0}$ & $6.5 \times 10^{1}$ & $2.6 \times 10^{1}$ & $3.2 \times 10^{1}$ & $2.2 \times 10^{1}$ & $3.2 \times 10^{0}$ & $8.6 \times 10^{-1}$ \\
\hline $05-10$ & 5 & $2.7 \times 10^{0}$ & $3.1 \times 10^{1}$ & $7.2 \times 10^{0}$ & $1.2 \times 10^{1}$ & $1.1 \times 10^{1}$ & $2.2 \times 10^{0}$ & $9.0 \times 10^{-1}$ \\
\hline $10-15$ & 5 & $1.6 \times 10^{0}$ & $4.8 \times 10^{1}$ & $8.6 \times 10^{0}$ & $1.6 \times 10^{1}$ & $1.9 \times 10^{1}$ & $2.1 \times 10^{0}$ & $1.3 \times 10^{0}$ \\
\hline $15-25$ & 5 & $2.7 \times 10^{0}$ & $3.7 \times 10^{1}$ & $4.6 \times 10^{0}$ & $1.2 \times 10^{1}$ & $1.5 \times 10^{1}$ & $2.0 \times 10^{0}$ & $1.1 \times 10^{0}$ \\
\hline $25-40$ & 5 & $1.1 \times 10^{0}$ & $7.1 \times 10^{0}$ & $3.7 \times 10^{0}$ & $3.8 \times 10^{0}$ & $2.2 \times 10^{0}$ & $1.2 \times 10^{0}$ & $6.8 \times 10^{-1}$ \\
\hline $40-60$ & 5 & $9.2 \times 10^{-1}$ & $3.2 \times 10^{0}$ & $2.6 \times 10^{0}$ & $2.2 \times 10^{0}$ & $1.1 \times 10^{0}$ & $6.7 \times 10^{-1}$ & $5.9 \times 10^{-1}$ \\
\hline $0-05$ & 5 & $6.4 \times 10^{0}$ & $6.5 \times 10^{1}$ & $2.6 \times 10^{1}$ & $3.2 \times 10^{1}$ & $2.2 \times 10^{1}$ & $3.2 \times 10^{0}$ & $8.6 \times 10^{-1}$ \\
\hline $0-10$ & 5 & $6.3 \times 10^{0}$ & $3.6 \times 10^{1}$ & $2.0 \times 10^{1}$ & $2.2 \times 10^{1}$ & $1.1 \times 10^{1}$ & $3.0 \times 10^{0}$ & $6.7 \times 10^{-1}$ \\
\hline $0-15$ & 5 & $1.4 \times 10^{1}$ & $2.6 \times 10^{1}$ & $2.0 \times 10^{1}$ & $2.0 \times 10^{1}$ & $5.2 \times 10^{0}$ & $3.0 \times 10^{0}$ & $2.7 \times 10^{-1}$ \\
\hline $0-25$ & 5 & $1.0 \times 10^{1}$ & $2.7 \times 10^{1}$ & $1.6 \times 10^{1}$ & $1.7 \times 10^{1}$ & $6.3 \times 10^{0}$ & $2.8 \times 10^{0}$ & $3.6 \times 10^{-1}$ \\
\hline $0-40$ & 5 & $9.0 \times 10^{0}$ & $1.9 \times 10^{1}$ & $1.0 \times 10^{1}$ & $1.2 \times 10^{1}$ & $3.8 \times 10^{0}$ & $2.4 \times 10^{0}$ & $2.8 \times 10^{-1}$ \\
\hline $0-60$ & 5 & $6.8 \times 10^{0}$ & $1.3 \times 10^{1}$ & $7.2 \times 10^{0}$ & $8.7 \times 10^{0}$ & $2.8 \times 10^{0}$ & $2.1 \times 10^{0}$ & $2.8 \times 10^{-1}$ \\
\hline
\end{tabular}

NOTE: Specific activity is decay corrected to 1994 .

$\mathrm{N}^{\mathrm{a}}$ stands for number of individual samples. 
Table C-10. Strontium-90 radionuclide concentration summary for all soil profiles taken during the 1978 NMIRS on Mellu Island (F23).

\begin{tabular}{|c|c|c|c|c|c|c|c|c|}
\hline \multirow{2}{*}{$\begin{array}{l}\text { Soil } \\
\text { depth } \\
\text { (cm) }\end{array}$} & \multirow[b]{2}{*}{$\mathrm{Na}$} & \multicolumn{5}{|c|}{ pCi g-1 dry wt } & \multirow{2}{*}{$\begin{array}{l}\text { Mean } \\
\text { of logs }\end{array}$} & \multirow{2}{*}{$\begin{array}{c}\mathrm{SD} \\
\text { of logs } \\
\end{array}$} \\
\hline & & Minimum & Maximum & Median & Mean & $\mathrm{SD}$ & & \\
\hline $0-05$ & 4 & $1.9 \times 10^{1}$ & $3.8 \times 10^{1}$ & $3.5 \times 10^{1}$ & $3.2 \times 10^{1}$ & $8.4 \times 10^{0}$ & $3.4 \times 10^{0}$ & $3.1 \times 10^{-1}$ \\
\hline $05-10$ & 4 & $8.6 \times 10^{0}$ & $3.1 \times 10^{1}$ & $2.7 \times 10^{1}$ & $2.3 \times 10^{1}$ & $9.8 \times 10^{0}$ & $3.0 \times 10^{0}$ & $5.9 \times 10^{=1}$ \\
\hline $10-15$ & 4 & $8.4 \times 10^{0}$ & $3.2 \times 10^{1}$ & $1.4 \times 10^{1}$ & $1.7 \times 10^{1}$ & $1.0 \times 10^{1}$ & $2.7 \times 10^{0}$ & $5.6 \times 10^{-1}$ \\
\hline $15-25$ & 4 & $4.6 \times 10^{0}$ & $2.3 \times 10^{1}$ & $1.2 \times 10^{1}$ & $1.3 \times 10^{1}$ & $8.1 \times 10^{0}$ & $2.4 \times 10^{0}$ & $7.0 \times 10^{-1}$ \\
\hline $25-40$ & 4 & $1.7 \times 10^{0}$ & $1.1 \times 10^{1}$ & $1.0 \times 10^{1}$ & $8.3 \times 10^{0}$ & $4.5 \times 10^{0}$ & $1.9 \times 10^{0}$ & $9.3 \times 10^{-1}$ \\
\hline $40-60$ & 1 & $2.3 \times 10^{0}$ & $2.3 \times 10^{0}$ & $2.3 \times 10^{0}$ & $2.3 \times 10^{0}$ & $0.0 \times 10^{0}$ & $8.4 \times 10^{-1}$ & $0.0 \times 10^{0}$ \\
\hline $0-05$ & 4 & $1.9 \times 10^{1}$ & $3.8 \times 10^{1}$ & $3.5 \times 10^{1}$ & $3.2 \times 10^{1}$ & $8.4 \times 10^{0}$ & $3.4 \times 10^{0}$ & $3.1 \times 10^{-1}$ \\
\hline $0-10$ & 4 & $1.4 \times 10^{1}$ & $3.4 \times 10^{1}$ & $3.1 \times 10^{1}$ & $2.7 \times 10^{1}$ & $9.1 \times 10^{0}$ & $3.3 \times 10^{0}$ & $4.2 \times 10^{-1}$ \\
\hline $0-15$ & 4 & $1.2 \times 10^{1}$ & $3.3 \times 10^{1}$ & $2.5 \times 10^{1}$ & $2.4 \times 10^{1}$ & $8.8 \times 10^{0}$ & $3.1 \times 10^{0}$ & $4.3 \times 10^{-1}$ \\
\hline $0-25$ & 4 & $1.1 \times 10^{1}$ & $2.9 \times 10^{1}$ & $1.9 \times 10^{1}$ & $2.0 \times 10^{1}$ & $7.8 \times 10^{0}$ & $2.9 \times 10^{0}$ & $4.2 \times 10^{-1}$ \\
\hline $0-40$ & 4 & $1.1 \times 10^{1}$ & $2.3 \times 10^{1}$ & $1.4 \times 10^{1}$ & $1.5 \times 10^{1}$ & $5.7 \times 10^{0}$ & $2.7 \times 10^{0}$ & $3.7 \times 10^{-1}$ \\
\hline $0-60$ & 1 & $1.2 \times 10^{1}$ & $1.2 \times 10^{1}$ & $1.2 \times 10^{1}$ & $1.2 \times 10^{1}$ & $0.0 \times 10^{0}$ & $2.5 \times 10^{0}$ & $0.0 \times 10^{0}$ \\
\hline
\end{tabular}

NOTE: Specific activity is decay corrected to 1994.

$\mathrm{N}^{\mathrm{a}}$ stands for number of individual samples. 
Table C-11. Plutonium-239+240 radionuclide concentration summary for all soil profiles taken during the 1978 NMIRS together with our recent trip in 1989 on Naen Island (F1).

\begin{tabular}{|c|c|c|c|c|c|c|c|c|}
\hline \multirow{2}{*}{$\begin{array}{l}\text { Soil } \\
\text { depth } \\
\text { (cm) }\end{array}$} & \multirow[b]{2}{*}{$\mathrm{Na}^{\mathrm{a}}$} & \multicolumn{5}{|c|}{ pCi $g^{-1}$ dry wt } & \multirow{2}{*}{$\begin{array}{r}\text { Mean } \\
\text { of logs }\end{array}$} & \multirow{2}{*}{$\begin{array}{c}\text { SD } \\
\text { of } \operatorname{logs} \\
\end{array}$} \\
\hline & & Minimum & Maximum & Median & Mean & $\mathrm{SD}$ & & \\
\hline $0-05$ & 16 & $4.8 \times 10^{0}$ & $1.0 \times 10^{2}$ & $4.9 \times 10^{1}$ & $4.3 \times 10^{1}$ & $2.5 \times 10^{1}$ & $3.5 \times 10^{0}$ & $8.1 \times 10^{-1}$ \\
\hline $05-10$ & 7 & $3.1 \times 10^{0}$ & $5.2 \times 10^{1}$ & $1.8 \times 10^{1}$ & $2.1 \times 10^{1}$ & $1.6 \times 10^{1}$ & $2.8 \times 10^{0}$ & $8.6 \times 10^{-1}$ \\
\hline $10-15$ & 7 & $2.1 \times 10^{0}$ & $1.9 \times 10^{1}$ & $8.2 \times 10^{0}$ & $1.1 \times 10^{1}$ & $6.4 \times 10^{0}$ & $2.2 \times 10^{0}$ & $7.7 \times 10^{-1}$ \\
\hline $15-25$ & 7 & $2.8 \times 10^{-1}$ & $7.6 \times 10^{0}$ & $1.4 \times 10^{0}$ & $2.2 \times 10^{0}$ & $2.6 \times 10^{0}$ & $2.5 \times 10^{-1}$ & $1.2 \times 10^{0}$ \\
\hline $25-40$ & 7 & $6.6 \times 10^{-2}$ & $3.3 \times 10^{0}$ & $1.4 \times 10^{-1}$ & $6.9 \times 10^{-1}$ & $1.2 \times 10^{0}$ & $-1.3 \times 10^{0}$ & $1.4 \times 10^{0}$ \\
\hline $40-60$ & 7 & $4.5 \times 10^{-2}$ & $5.5 \times 10^{-1}$ & $1.8 \times 10^{-1}$ & $2.0 \times 10^{-1}$ & $1.7 \times 10^{-1}$ & $-1.9 \times 10^{0}$ & $8.5 \times 10^{-1}$ \\
\hline $0-05$ & 16 . & $4.8 \times 10^{0}$ & $1.0 \times 10^{2}$ & $4.9 \times 10^{1}$ & $4.3 \times 10^{1}$ & $2.5 \times 10^{1}$ & $3.5 \times 10^{0}$ & $8.1 \times 10^{-1}$ \\
\hline $0-10$ & 7 & $8.4 \times 10^{0}$ & $5.7 \times 10^{1}$ & $2.3 \times 10^{1}$ & $2.5 \times 10^{1}$ & $1.7 \times 10^{1}$ & $3.0 \times 10^{0}$ & $6.6 \times 10^{-1}$ \\
\hline $0-15$ & 7 & $8.3 \times 10^{0}$ & $4.4 \times 10^{1}$ & $1.8 \times 10^{1}$ & $2.0 \times 10^{1}$ & $1.2 \times 10^{1}$ & $2.9 \times 10^{0}$ & $5.8 \times 10^{-1}$ \\
\hline $0-25$ & 7 & $5.2 \times 10^{0}$ & $2.7 \times 10^{1}$ & $1.1 \times 10^{1}$ & $1.3 \times 10^{1}$ & $7.4 \times 10^{0}$ & $2.4 \times 10^{0}$ & $5.6 \times 10^{-1}$ \\
\hline $0-40$ & 7 & $3.3 \times 10^{0}$ & $1.7 \times 10^{1}$ & $6.8 \times 10^{0}$ & $8.4 \times 10^{0}$ & $4.7 \times 10^{0}$ & $2.0 \times 10^{0}$ & $5.7 \times 10^{-1}$ \\
\hline $0-60$ & 7 & $2.2 \times 10^{0}$ & $1.1 \times 10^{1}$ & $4.5 \times 10^{0}$ & $5.7 \times 10^{0}$ & $3.2 \times 10^{0}$ & $1.6 \times 10^{0}$ & $5.7 \times 10^{-1}$ \\
\hline
\end{tabular}

NOTE: Specific activity is decay corrected to 1994 .

$\mathrm{N}^{a}$ stands for number of individual samples. 
Table C-12. Plutonium-239+240 radionuclide concentration summary for all soil profiles taken during the 1978 NMIRS together with our recent trip in 1989 on Yugui Island (F5).

\begin{tabular}{|c|c|c|c|c|c|c|c|c|}
\hline \multirow{2}{*}{$\begin{array}{l}\text { Soil } \\
\text { depth } \\
\text { (cm) }\end{array}$} & \multirow[b]{2}{*}{$\mathrm{Na}^{\mathrm{a}}$} & \multicolumn{5}{|c|}{ pCi $g^{-1}$ dry wt } & \multirow{2}{*}{$\begin{array}{l}\text { Mean } \\
\text { of logs }\end{array}$} & \multirow{2}{*}{$\begin{array}{c}\text { SD } \\
\text { of logs }\end{array}$} \\
\hline & & Minimum & Maximum & Median & Mean & $\overline{\mathrm{SD}}$ & & \\
\hline $0-05$ & 11 & $1.5 \times 10^{1}$ & $1.2 \times 10^{2}$ & $5.5 \times 10^{1}$ & $5.8 \times 10^{1}$ & $3.1 \times 10^{1}$ & $3.9 \times 10^{0}$ & $5.9 \times 10^{-1}$ \\
\hline $05-10$ & 1 & $2.3 \times 10^{0}$ & $2.3 \times 10^{0}$ & $2.3 \times 10^{0}$ & $2.3 \times 10^{0}$ & $0.0 \times 10^{0}$ & $8.1 \times 10^{-1}$ & $0.0 \times 10^{0}$ \\
\hline $10-15$ & 1 & $6.6 \times 10^{-1}$ & $6.6 \times 10^{-1}$ & $6.6 \times 10^{-1}$ & $6.6 \times 10^{-1}$ & $0.0 \times 10^{0}$ & $-4.1 \times 10^{-1}$ & $0.0 \times 10^{0}$ \\
\hline $15-25$ & 1 & $2.3 \times 10^{-1}$ & $2.3 \times 10^{-1}$ & $2.3 \times 10^{-1}$ & $2.3 \times 10^{-1}$ & $0.0 \times 10^{0}$ & $-1.5 \times 10^{0}$ & $0.0 \times 10^{0}$ \\
\hline $25-40$ & 1 & $1.2 \times 10^{-1}$ & $1.2 \times 10^{-1}$ & $1.2 \times 10^{-1}$ & $1.2 \times 10^{-1}$ & $0.0 \times 10^{0}$ & $-2.2 \times 10^{0}$ & $0.0 \times 10^{0}$ \\
\hline $40-60$ & 1 & $3.2 \times 10^{-2}$ & $3.2 \times 10^{-2}$ & $3.2 \times 10^{-2}$ & $3.2 \times 10^{-2}$ & $0.0 \times 10^{0}$ & $-3.4 \times 10^{0}$ & $0.0 \times 10^{0}$ \\
\hline $0-05$ & 11 & $1.5 \times 10^{1}$ & $1.2 \times 10^{2}$ & $5.5 \times 10^{1}$ & $5.8 \times 10^{1}$ & $3.1 \times 10^{1}$ & $3.9 \times 10^{0}$ & $5.9 \times 10^{-1}$ \\
\hline $0-10$ & 1 & $8.6 \times 10^{0}$ & $8.6 \times 10^{0}$ & $8.6 \times 10^{0}$ & $8.6 \times 10^{\circ}$ & $0.0 \times 10^{0}$ & $2.2 \times 10^{0}$ & $0.0 \times 10^{0}$ \\
\hline $0-15$ & 1 & $5.9 \times 10^{0}$ & $5.9 \times 10^{0}$ & $5.9 \times 10^{0}$ & $5.9 \times 10^{0}$ & $0.0 \times 10^{0}$ & $1.8 \times 10^{0}$ & $0.0 \times 10^{0}$ \\
\hline $0-25$ & 1 & $3.7 \times 10^{0}$ & $3.7 \times 10^{0}$ & $3.7 \times 10^{0}$ & $3.7 \times 10^{0}$ & $0.0 \times 10^{0}$ & $1.3 \times 10^{0}$ & $0.0 \times 10^{0}$ \\
\hline $0-40$ & 1 & $2.3 \times 10^{0}$ & $2.3 \times 10^{0}$ & $2.3 \times 10^{0}$ & $2.3 \times 10^{0}$ & $0.0 \times 10^{0}$ & $8.5 \times 10^{-1}$ & $0.0 \times 10^{0}$ \\
\hline $0-60$ & 1 & $1.6 \times 10^{0}$ & $1.6 \times 10^{0}$ & $1.6 \times 10^{0}$ & $1.6 \times 10^{0}$ & $0.0 \times 10^{0}$ & $4.5 \times 10^{-1}$ & $0.0 \times 10^{0}$ \\
\hline
\end{tabular}

NOTE: Specific activity is decay corrected to 1994.

$\mathrm{N}^{\mathrm{a}}$ stands for number of individual samples. 
Table C-13. Plutonium-239+240 radionuclide concentration summary for all soil profiles taken during the 1978 NMIRS together with our recent trip in 1989 on Lomiulal Island (F7).

\begin{tabular}{|c|c|c|c|c|c|c|c|c|}
\hline \multirow{2}{*}{$\begin{array}{l}\text { Soil } \\
\text { depth } \\
(\mathrm{cm})\end{array}$} & \multirow[b]{2}{*}{$\mathrm{Na}$} & \multicolumn{5}{|c|}{ pCi $g^{-1}$ dry wt } & \multirow{2}{*}{$\begin{array}{l}\text { Mean } \\
\text { of logs } \\
\end{array}$} & \multirow{2}{*}{$\begin{array}{c}\text { SD } \\
\text { of logs } \\
\end{array}$} \\
\hline & & Minimum & Maximum & Median & Mean & $\overline{S D}$ & & \\
\hline $0-05$ & 14 & $8.6 \times 10^{0}$ & $6.7 \times 10^{1}$ & $3.5 \times 10^{1}$ & $3.4 \times 10^{1}$ & $1.8 \times 10^{1}$ & $3.4 \times 10^{0}$ & $6.5 \times 10^{-1}$ \\
\hline $05-10$ & 4 & $3.9 \times 10^{0}$ & $1.7 \times 10^{1}$ & $1.6 \times 10^{1}$ & $1.3 \times 10^{1}$ & $6.2 \times 10^{0}$ & $2.4 \times 10^{0}$ & $7.1 \times 10^{-1}$ \\
\hline $10-15$ & 4 & $1.4 \times 10^{0}$ & $1.5 \times 10^{1}$ & $4.4 \times 10^{0}$ & $6.3 \times 10^{0}$ & $6.1 \times 10^{0}$ & $1.5 \times 10^{0}$ & $1.0 \times 10^{0}$ \\
\hline $15-25$ & 4 & $5.4 \times 10^{-1}$ & $6.3 \times 10^{0}$ & $1.1 \times 10^{0}$ & $2.3 \times 10^{0}$ & $2.7 \times 10^{0}$ & $3.6 \times 10^{-1}$ & $1.1 \times 10^{0}$ \\
\hline $25-40$ & 4 & $8.6 \times 10^{-2}$ & $7.2 \times 10^{-1}$ & $4.1 \times 10^{-1}$ & $4.0 \times 10^{-1}$ & $3.2 \times 10^{-1}$ & $-1.2 \times 10^{0}$ & $1.0 \times 10^{0}$ \\
\hline $40-60$ & 4 & $1.9 \times 10^{-2}$ & $6.3 \times 10^{-1}$ & $1.3 \times 10^{-1}$ & $2.3 \times 10^{-1}$ & $2.8 \times 10^{-1}$ & $-2.2 \times 10^{0}$ & $1.5 \times 10^{0}$ \\
\hline $0-05$ & 14 & $8.6 \times 10^{0}$ & $6.7 \times 10^{1}$ & $3.5 \times 10^{1}$ & $3.4 \times 10^{1}$ & $1.8 \times 10^{1}$ & $3.4 \times 10^{0}$ & $6.5 \times 10^{-1}$ \\
\hline $0-10$ & 4 & $1.3 \times 10^{1}$ & $2.1 \times 10^{1}$ & $1.6 \times 10^{1}$ & $1.6 \times 10^{1}$ & $3.8 \times 10^{0}$ & $2.8 \times 10^{0}$ & $2.4 \times 10^{-1}$ \\
\hline $0-15$ & 4 & $1.1 \times 10^{1}$ & $1.5 \times 10^{1}$ & $1.3 \times 10^{1}$ & $1.3 \times 10^{1}$ & $1.8 \times 10^{0}$ & $2.5 \times 10^{0}$ & $1.4 \times 10^{-1}$ \\
\hline $0-25$ & 4 & $6.9 \times 10^{0}$ & $1.1 \times 10^{1}$ & $8.4 \times 10^{0}$ & $8.6 \times 10^{0}$ & $1.7 \times 10^{0}$ & $2.1 \times 10^{0}$ & $1.9 \times 10^{-1}$ \\
\hline $0-40$ & 4 & $4.4 \times 10^{0}$ & $6.9 \times 10^{0}$ & $5.4 \times 10^{0}$ & $5.5 \times 10^{0}$ & $1.1 \times 10^{0}$ & $1.7 \times 10^{0}$ & $1.9 \times 10^{-1}$ \\
\hline $0-60$ & 4 & $2.9 \times 10^{0}$ & $4.8 \times 10^{0}$ & $3.7 \times 10^{0}$ & $3.8 \times 10^{0}$ & $7.9 \times 10^{-1}$ & $1.3 \times 10^{0}$ & $2.1 \times 10^{-1}$ \\
\hline
\end{tabular}

NOTE: Specific activity is decay corrected to 1994 .

$\mathrm{N}^{\mathrm{a}}$ stands for number of individual samples. 
Table C-14. Plutonium-239+240 radionuclide concentration summary for all soil profiles taken during the 1978 NMIRS on Kabelle Island (F13).

\begin{tabular}{|c|c|c|c|c|c|c|c|c|}
\hline \multirow{2}{*}{$\begin{array}{r}\text { Soil } \\
\text { depth } \\
(\mathrm{cm})\end{array}$} & \multirow[b]{2}{*}{$\mathrm{Na}^{\mathrm{a}}$} & \multicolumn{5}{|c|}{ pCi g-1 dry wt } & \multirow{2}{*}{$\begin{array}{c}\text { Mean } \\
\text { of logs }\end{array}$} & \multirow{2}{*}{$\begin{array}{c}\text { SD } \\
\text { of logs } \\
\end{array}$} \\
\hline & & Minimum & Maximum & Median & Mean & $\overline{\mathrm{SD}}$ & & \\
\hline $0-05$ & 5 & $5.5 \times 10^{-1}$ & $3.2 \times 10^{1}$ & $8.3 \times 10^{0}$ & $1.4 \times 10^{1}$ & $1.5 \times 10^{1}$ & $1.8 \times 10^{0}$ & $1.8 \times 10^{0}$ \\
\hline $05-10$ & 5 & $5.0 \times 10^{-1}$ & $7.8 \times 10^{0}$ & $1.6 \times 10^{0}$ & $3.1 \times 10^{0}$ & $2.9 \times 10^{0}$ & $7.4 \times 10^{-1}$ & $1.1 \times 10^{0}$ \\
\hline $10-15$ & 5 & $4.5 \times 10^{-1}$ & $1.2 \times 10^{1}$ & $6.8 \times 10^{-1}$ & $3.6 \times 10^{0}$ & $4.8 \times 10^{0}$ & $4.7 \times 10^{-1}$ & $1.4 \times 10^{0}$ \\
\hline $15-25$ & 5 & $1.8 \times 10^{-1}$ & $9.4 \times 10^{0}$ & $1.7 \times 10^{0}$ & $2.9 \times 10^{0}$ & $3.8 \times 10^{0}$ & $2.0 \times 10^{-1}$ & $1.6 \times 10^{0}$ \\
\hline $25-40$ & 5 & $7.1 \times 10^{-2}$ & $1.8 \times 10^{0}$ & $3.6 \times 10^{-1}$ & $7.1 \times 10^{-1}$ & $7.0 \times 10^{-1}$ & $-8.7 \times 10^{-1}$ & $1.3 \times 10^{0}$ \\
\hline $40-60$ & 5 & $5.1 \times 10^{-2}$ & $5.0 \times 10^{-1}$ & $1.2 \times 10^{-1}$ & $2.3 \times 10^{-1}$ & $2.0 \times 10^{-1}$ & $-1.8 \times 10^{0}$ & $9.9 \times 10^{-1}$ \\
\hline $0-05$ & 5 & $5.5 \times 10^{-1}$ & $3.2 \times 10^{1}$ & $8.3 \times 10^{0}$ & $1.4 \times 10^{1}$ & $1.5 \times 10^{1}$ & $1.8 \times 10^{0}$ & $1.8 \times 10^{0}$ \\
\hline $0-10$ & 5 & $5.3 \times 10^{-1}$ & $1.7 \times 10^{1}$ & $8.0 \times 10^{0}$ & $8.7 \times 10^{0}$ & $7.7 \times 10^{0}$ & $1.5 \times 10^{0}$ & $1.5 \times 10^{0}$ \\
\hline $0-15$ & 5 & $5.8 \times 10^{-1}$ & $1.2 \times 10^{1}$ & $6.8 \times 10^{0}$ & $7.0 \times 10^{0}$ & $4.5 \times 10^{0}$ & $1.6 \times 10^{0}$ & $1.2 \times 10^{0}$ \\
\hline $0-25$ & 5 & $1.0 \times 10^{0}$ & $7.6 \times 10^{0}$ & $6.7 \times 10^{0}$ & $5.3 \times 10^{0}$ & $2.7 \times 10^{0}$ & $1.5 \times 10^{0}$ & $8.5 \times 10^{-1}$ \\
\hline $0-40$ & 5 & $1.3 \times 10^{0}$ & $4.9 \times 10^{0}$ & $4.4 \times 10^{0}$ & $3.6 \times 10^{0}$ & $1.5 \times 10^{0}$ & $1.2 \times 10^{0}$ & $5.6 \times 10^{-1}$ \\
\hline $0-60$ & 5 & $1.0 \times 10^{0}$ & $3.3 \times 10^{0}$ & $3.0 \times 10^{0}$ & $2.5 \times 10^{0}$ & $9.9 \times 10^{-1}$ & $8.2 \times 10^{-1}$ & $5.0 \times 10^{-1}$ \\
\hline
\end{tabular}

NOTE: Specific activity is decay corrected to 1994.

$\mathrm{N}^{\mathrm{a}}$ stands for number of individual samples. 
Table C-15. Plutonium-239+240 radionuclide concentration summary for all soil profiles taken during the 1978 NMIRS together with our recent trip in 1989 on Mellu Island (F23).

\begin{tabular}{|c|c|c|c|c|c|c|c|c|}
\hline $\begin{array}{l}\text { Soil } \\
\text { depth }\end{array}$ & & & & $i g^{-1}$ dry & & & Mean & SD \\
\hline$(\mathrm{cm})$ & $\mathrm{Na}$ & Minimum & Maximum & Median & Mean & SD & of $\log s$ & of $\log s$ \\
\hline $0-05$ & 15 & $3.0 \times 10^{0}$ & $2.3 \times 10^{1}$ & $1.7 \times 10^{1}$ & $1.4 \times 10^{1}$ & $7.1 \times 10^{0}$ & $2.4 \times 10^{0}$ & $6.9 \times 10^{-1}$ \\
\hline $05-10$ & 4 & $2.5 \times 10^{0}$ & $1.2 \times 10^{1}$ & $3.5 \times 10^{0}$ & $5.3 \times 10^{0}$ & $4.3 \times 10^{0}$ & $1.5 \times 10^{0}$ & $6.9 \times 10^{-1}$ \\
\hline $10-15$ & 4 & $7.2 \times 10^{-1}$ & $8.7 \times 10^{0}$ & $2.6 \times 10^{0}$ & $3.6 \times 10^{0}$ & $3.6 \times 10^{0}$ & $9.0 \times 10^{-1}$ & $1.1 \times 10^{0}$ \\
\hline $15-25$ & 4 & $3.6 \times 10^{-1}$ & $3.2 \times 10^{0}$ & $1.3 \times 10^{0}$ & $1.5 \times 10^{0}$ & $1.3 \times 10^{0}$ & $1.1 \times 10^{-1}$ & $9.7 \times 10^{-1}$ \\
\hline $25-40$ & 4 & $1.7 \times 10^{-1}$ & $2.2 \times 10^{0}$ & $1.1 \times 10^{0}$ & $1.1 \times 10^{0}$ & $9.8 \times 10^{-1}$ & $-3.3 \times 10^{-1}$ & $1.2 \times 10^{0}$ \\
\hline $40-60$ & 1 & $6.4 \times 10^{-2}$ & $6.4 \times 10^{-2}$ & $6.4 \times 10^{-2}$ & $6.4 \times 10^{-2}$ & $0.0 \times 10^{0}$ & $-2.8 \times 10^{0}$ & $0.0 \times 10^{0}$ \\
\hline $0-05$ & 15 & $3.0 \times 10^{0}$ & $2.3 \times 10^{1}$ & $1.7 \times 10^{1}$ & $1.4 \times 10^{1}$ & $7.1 \times 10^{0}$ & $2.4 \times 10^{0}$ & $6.9 \times 10^{-1}$ \\
\hline $0-10$ & 4 & $5.4 \times 10^{0}$ & $1.4 \times 10^{1}$ & $9.9 \times 10^{0}$ & $9.9 \times 10^{0}$ & $3.7 \times 10^{0}$ & $2.2 \times 10^{0}$ & $4.1 \times 10^{-1}$ \\
\hline $0-15$ & 4 & $4.7 \times 10^{0}$ & $1.2 \times 10^{1}$ & $7.0 \times 10^{0}$ & $7.8 \times 10^{0}$ & $3.3 \times 10^{0}$ & $2.0 \times 10^{0}$ & $4.0 \times 10^{-1}$ \\
\hline $0-25$ & 4 & $3.6 \times 10^{0}$ & $8.7 \times 10^{0}$ & $4.4 \times 10^{0}$ & $5.3 \times 10^{0}$ & $2.3 \times 10^{0}$ & $1.6 \times 10^{0}$ & $3.9 \times 10^{-1}$ \\
\hline $0-40$ & 4 & $2.7 \times 10^{0}$ & $6.1 \times 10^{0}$ & $3.0 \times 10^{0}$ & $3.7 \times 10^{0}$ & $1.6 \times 10^{0}$ & $1.3 \times 10^{0}$ & $3.7 \times 10^{-1}$ \\
\hline $0-60$ & 1. & $1.8 \times 10^{0}$ & $1.8 \times 10^{0}$ & $1.8 \times 10^{0}$ & $1.8 \times 10^{0}$ & $0.0 \times 10^{0}$ & $6.1 \times 10^{-1}$ & $0.0 \times 10^{0}$ \\
\hline
\end{tabular}

NOTE: Specific activity is decay corrected to 1994 .

$\mathrm{N}^{\mathrm{a}}$ stands for number of individual samples. 
Table C-16. Americium-241 radionuclide concentration summary for all soil profiles taken from the 1978 NMIRS together with our most recent trip in 1989 on Naen Island (F1).

\begin{tabular}{|c|c|c|c|c|c|c|c|c|}
\hline \multirow{2}{*}{$\begin{array}{c}\text { Soil } \\
\text { depth } \\
\text { (cm) }\end{array}$} & \multirow[b]{2}{*}{$\mathrm{Na}^{\mathrm{a}}$} & \multicolumn{5}{|c|}{ pCi g $^{-1}$ dry wt } & \multirow{2}{*}{$\begin{array}{r}\text { Mean } \\
\text { of logs }\end{array}$} & \multirow{2}{*}{$\begin{array}{c}\text { SD } \\
\text { of logs }\end{array}$} \\
\hline & & Minimum & Maximum & Median & Mean & $\mathrm{SD}$ & & \\
\hline $0-05$ & 16 & $6.9 \times 10^{0}$ & $6.9 \times 10^{1}$ & $2.6 \times 10^{1}$ & $2.9 \times 10^{1}$ & $2.0 \times 10^{1}$ & $3.1 \times 10^{0}$ & $7.8 \times 10^{-1}$ \\
\hline $05-10$ & 15 & $1.3 \times 10^{0}$ & $1.5 \times 10^{1}$ & $8.7 \times 10^{0}$ & $8.5 \times 10^{0}$ & $4.2 \times 10^{0}$ & $2.0 \times 10^{0}$ & $6.6 \times 10^{-1}$ \\
\hline $10-15$ & 14 & $2.5 \times 10^{-1}$ & $1.1 \times 10^{1}$ & $3.3 \times 10^{0}$ & $3.3 \times 10^{0}$ & $2.9 \times 10^{0}$ & $7.1 \times 10^{-1}$ & $1.1 \times 10^{0}$ \\
\hline $15-25$ & 10 & $1.0 \times 10^{-1}$ & $3.9 \times 10^{0}$ & $6.3 \times 10^{-1}$ & $1.1 \times 10^{0}$ & $1.1 \times 10^{0}$ & $-4.2 \times 10^{-1}$ & $1.1 \times 10^{0}$ \\
\hline $25-40$ & 9 & $3.0 \times 10^{-2}$ & $2.0 \times 10^{0}$ & $1.2 \times 10^{-1}$ & $3.1 \times 10^{-1}$ & $6.3 \times 10^{-1}$ & $-2.2 \times 10^{0}$ & $1.3 \times 10^{0}$ \\
\hline $40-60$ & 6 & $1.4 \times 10^{-2}$ & $3.6 \times 10^{-1}$ & $6.9 \times 10^{-2}$ & $1.1 \times 10^{-1}$ & $1.3 \times 10^{-1}$ & $-2.8 \times 10^{0}$ & $1.2 \times 10^{0}$ \\
\hline $0-05$ & 16 & $6.9 \times 10^{0}$ & $6.9 \times 10^{1}$ & $2.6 \times 10^{1}$ & $2.9 \times 10^{1}$ & $2.0 \times 10^{1}$ & $3.1 \times 10^{0}$ & $7.8 \times 10^{-1}$ \\
\hline $0-10$ & 15 & $6.1 \times 10^{0}$ & $3.7 \times 10^{1}$ & $2.1 \times 10^{1}$ & $1.9 \times 10^{1}$ & $9.6 \times 10^{0}$ & $2.8 \times 10^{0}$ & $5.5 \times 10^{-1}$ \\
\hline $0-15$ & 13 & $4.3 \times 10^{0}$ & $2.2 \times 10^{1}$ & $1.2 \times 10^{1}$ & $1.3 \times 10^{1}$ & $5.7 \times 10^{0}$ & $2.5 \times 10^{0}$ & $4.9 \times 10^{-1}$ \\
\hline $0-25$ & 10 & $2.7 \times 10^{0}$ & $1.4 \times 10^{1}$ & $6.8 \times 10^{0}$ & $7.1 \times 10^{0}$ & $3.2 \times 10^{0}$ & $1.9 \times 10^{0}$ & $4.7 \times 10^{-1}$ \\
\hline $0-40$ & 8 & $1.7 \times 10^{0}$ & $8.5 \times 10^{0}$ & $4.3 \times 10^{0}$ & $4.6 \times 10^{0}$ & $2.2 \times 10^{0}$ & $1.4 \times 10^{0}$ & $5.2 \times 10^{-1}$ \\
\hline $0-60$ & 6 & $1.1 \times 10^{\circ}$ & $4.3 \times 10^{0}$ & $2.4 \times 10^{0}$ & $2.5 \times 10^{0}$ & $1.1 \times 10^{\circ}$ & $8.4 \times 10^{-1}$ & $4.6 \times 10^{-1}$ \\
\hline
\end{tabular}

NOTE: Specific activity is decay corrected to 1994.

$\mathrm{N}^{\mathrm{a}}$ stands for number of individual samples. 
Table C-17. Americium-241 radionuclide concentration summary for all soil profiles taken from the 1978 NMIRS together with our most recent trip in 1989 on Yugui Island (F5).

\begin{tabular}{|c|c|c|c|c|c|c|c|c|}
\hline \multirow{2}{*}{$\begin{array}{l}\text { Soil } \\
\text { depth } \\
\text { (cm) }\end{array}$} & \multirow[b]{2}{*}{$\mathrm{Na}^{\mathrm{a}}$} & \multicolumn{5}{|c|}{ pCi g-1 dry wt } & \multirow{2}{*}{$\begin{array}{l}\text { Mean } \\
\text { of logs }\end{array}$} & \multirow{2}{*}{$\begin{array}{r}\text { SD } \\
\text { of logs }\end{array}$} \\
\hline & & Minimum & Maximum & Median & Mean & $\mathrm{SD}$ & & \\
\hline $0-05$ & 11 & $6.5 \times 10^{0}$ & $1.2 \times 10^{2}$ & $4.8 \times 10^{1}$ & $5.3 \times 10^{1}$ & $3.1 \times 10^{1}$ & $3.8 \times 10^{0}$ & $7.7 \times 10^{-1}$ \\
\hline $05-10$ & 9 & $1.5 \times 10^{0}$ & $1.1 \times 10^{2}$ & $4.3 \times 10^{0}$ & $1.6 \times 10^{1}$ & $3.4 \times 10^{1}$ & $1.7 \times 10^{0}$ & $1.3 \times 10^{0}$ \\
\hline $10-15$ & 7 & $2.4 \times 10^{-1}$ & $4.9 \times 10^{0}$ & $8.9 \times 10^{-1}$ & $1.7 \times 10^{0}$ & $1.6 \times 10^{0}$ & $1.3 \times 10^{-1}$ & $1.0 \times 10^{0}$ \\
\hline $15-25$ & 4 & $7.7 \times 10^{-2}$ & $8.7 \times 10^{-1}$ & $3.2 \times 10^{-1}$ & $4.0 \times 10^{-1}$ & $3.4 \times 10^{-1}$ & $-1.3 \times 10^{0}$ & $1.0 \times 10^{0}$ \\
\hline $25-40$ & 1 & $5.4 \times 10^{-2}$ & $5.4 \times 10^{-2}$ & $5.4 \times 10^{-2}$ & $5.4 \times 10^{-2}$ & $0.0 \times 10^{0}$ & $-2.9 \times 10^{0}$ & $0.0 \times 10^{0}$ \\
\hline $40-60$ & 0 & $0.0 \times 10^{0}$ & $0.0 \times 10^{0}$ & $0.0 \times 10^{0}$ & $0.0 \times 10^{0}$ & $0.0 \times 10^{0}$ & $0.0 \times 10^{0}$ & $0.0 \times 10^{0}$ \\
\hline $0-05$ & 11 & $6.5 \times 10^{0}$ & $1.2 \times 10^{2}$ & $4.8 \times 10^{1}$ & $5.3 \times 10^{1}$ & $3.1 \times 10^{1}$ & $3.8 \times 10^{0}$ & $7.7 \times 10^{-1}$ \\
\hline $0-10$ & 9 & $4.0 \times 10^{0}$ & $8.3 \times 10^{1}$ & $2.7 \times 10^{1}$ & $3.4 \times 10^{1}$ & $2.5 \times 10^{1}$ & $3.2 \times 10^{0}$ & $8.8 \times 10^{-1}$ \\
\hline $0-15$ & 7 & $2.8 \times 10^{0}$ & $5.6 \times 10^{1}$ & $1.9 \times 10^{1}$ & $2.1 \times 10^{1}$ & $1.7 \times 10^{1}$ & $2.7 \times 10^{0}$ & $9.3 \times 10^{-1}$ \\
\hline $0-25$ & 2 & $1.7 \times 10^{0}$ & $3.4 \times 10^{1}$ & $1.8 \times 10^{1}$ & $1.8 \times 10^{1}$ & $2.3 \times 10^{1}$ & $2.0 \times 10^{0}$ & $2.1 \times 10^{0}$ \\
\hline $0-40$ & 1 & $1.1 \times 10^{0}$ & $1.1 \times 10^{0}$ & $1.1 \times 10^{0}$ & $1.1 \times 10^{0}$ & $0.0 \times 10^{0}$ & $7.6 \times 10^{-2}$ & $0.0 \times 10^{0}$ \\
\hline $0-60$ & 0 & $0.0 \times 10^{0}$ & $0.0 \times 10^{0}$ & $0.0 \times 10^{0}$ & $0.0 \times 10^{0}$ & $0.0 \times 10^{0}$ & $0.0 \times 10^{0}$ & $0.0 \times 10^{0}$ \\
\hline
\end{tabular}

NOTE: Specific activity is decay corrected to 1994.

$\mathrm{N}^{\mathrm{a}}$ stands for number of individual samples. 
Table C-18. Americium-241 radionuclide concentration summary for all soil profiles taken from the 1978 NMIRS together with our most recent trip in 1989 on Lomiulal Island (F7).

\begin{tabular}{|c|c|c|c|c|c|c|c|c|}
\hline \multirow{2}{*}{$\begin{array}{c}\text { Soil } \\
\text { depth } \\
(\mathrm{cm}) \\
\end{array}$} & \multirow[b]{2}{*}{$\mathrm{Na}^{\mathrm{a}}$} & \multicolumn{5}{|c|}{ pCi g-1 dry wt } & \multirow{2}{*}{$\begin{array}{l}\text { Mean } \\
\text { of logs }\end{array}$} & \multirow{2}{*}{$\begin{array}{c}\text { SD } \\
\text { of } \operatorname{logs} \\
\end{array}$} \\
\hline & & Minimum & Maximum & Median & Mean & $\mathrm{SD}$ & & \\
\hline $0-05$ & 12 & $4.7 \times 10^{0}$ & $4.5 \times 10^{1}$ & $2.2 \times 10^{1}$ & $2.2 \times 10^{1}$ & $1.3 \times 10^{1}$ & $2.9 \times 10^{0}$ & $7.6 \times 10^{-1}$ \\
\hline $05-10$ & 11 & $1.9 \times 10^{0}$ & $1.7 \times 10^{1}$ & $4.9 \times 10^{0}$ & $6.3 \times 10^{0}$ & $4.2 \times 10^{0}$ & $1.7 \times 10^{0}$ & $6.0 \times 10^{-1}$ \\
\hline $10-15$ & 9 & $1.2 \times 10^{0}$ & $8.9 \times 10^{0}$ & $2.3 \times 10^{0}$ & $3.0 \times 10^{0}$ & $2.4 \times 10^{0}$ & $8.9 \times 10^{-1}$ & $6.3 \times 10^{-1}$ \\
\hline $15-25$ & 7 & $2.0 \times 10^{-1}$ & $3.6 \times 10^{0}$ & $3.7 \times 10^{-1}$ & $8.4 \times 10^{-1}$ & $1.2 \times 10^{0}$ & $-7.1 \times 10^{-1}$ & $9.7 \times 10^{-1}$ \\
\hline $25-40$ & 3 & $5.6 \times 10^{-2}$ & $2.9 \times 10^{-1}$ & $1.9 \times 10^{-1}$ & $1.8 \times 10^{-1}$ & $1.2 \times 10^{-1}$ & $-1.9 \times 10^{0}$ & $8.5 \times 10^{-1}$ \\
\hline $40-60$ & 2 & $1.4 \times 10^{-2}$ & $3.4 \times 10^{-1}$ & $1.8 \times 10^{-1}$ & $1.8 \times 10^{-1}$ & $2.3 \times 10^{-1}$ & $-2.7 \times 10^{0}$ & $2.3 \times 10^{0}$ \\
\hline $0-05$ & 12 & $4.7 \times 10^{0}$ & $4.5 \times 10^{1}$ & $2.2 \times 10^{1}$ & $2.2 \times 10^{1}$ & $1.3 \times 10^{1}$ & $2.9 \times 10^{0}$ & $7.6 \times 10^{-1}$ \\
\hline $0-10$ & 11 & $6.1 \times 10^{0}$ & $2.4 \times 10^{1}$ & $1.3 \times 10^{1}$ & $1.4 \times 10^{1}$ & $6.4 \times 10^{0}$ & $2.5 \times 10^{0}$ & $5.0 \times 10^{-1}$ \\
\hline $0-15$ & 9 & $4.6 \times 10^{0}$ & $1.5 \times 10^{1}$ & $8.1 \times 10^{0}$ & $9.3 \times 10^{0}$ & $3.8 \times 10^{0}$ & $2.2 \times 10^{0}$ & $4.2 \times 10^{-1}$ \\
\hline $0-25$ & 4 & $3.4 \times 10^{0}$ & $8.6 \times 10^{0}$ & $5.5 \times 10^{0}$ & $5.7 \times 10^{0}$ & $2.3 \times 10^{0}$ & $1.7 \times 10^{0}$ & $4.0 \times 10^{-1}$ \\
\hline $0-40$ & 2 & $2.1 \times 10^{0}$ & $4.0 \times 10^{0}$ & $3.1 \times 10^{0}$ & $3.1 \times 10^{0}$ & $1.3 \times 10^{0}$ & $1.1 \times 10^{0}$ & $4.5 \times 10^{-1}$ \\
\hline $0-60$ & 2 & $1.4 \times 10^{0}$ & $2.8 \times 10^{0}$ & $2.1 \times 10^{0}$ & $2.1 \times 10^{0}$ & $9.6 \times 10^{-1}$ & $6.9 \times 10^{-1}$ & $4.7 \times 10^{-1}$ \\
\hline
\end{tabular}

NOTE: Specific activity is decay corrected to 1994.

$\mathrm{N}^{\mathrm{a}}$ stands for number of individual samples. 
Table C-19. Americium-241 radionuclide concentration summary for all soil profiles taken from the 1978 NMIRS together with our most recent trips in 1988 through 1989 on Kabelle Island (F13).

\begin{tabular}{|c|c|c|c|c|c|c|c|c|}
\hline \multirow{2}{*}{$\begin{array}{c}\text { Soil } \\
\text { depth } \\
\text { (cm) }\end{array}$} & \multirow[b]{2}{*}{$\mathrm{Na}$} & \multicolumn{5}{|c|}{ pCi $g^{-1}$ dry wt } & \multirow{2}{*}{$\begin{array}{c}\text { Mean } \\
\text { of logs }\end{array}$} & \multirow{2}{*}{$\begin{array}{c}\text { SD } \\
\text { of } \operatorname{logs} \\
\end{array}$} \\
\hline & & Minimum & Maximum & Median & Mean & $\overline{\mathrm{SD}}$ & & \\
\hline $0-05$ & 31 & $3.0 \times 10^{-1}$ & $3.0 \times 10^{1}$ & $1.1 \times 10^{1}$ & $1.2 \times 10^{1}$ & $6.9 \times 10^{0}$ & $2.2 \times 10^{0}$ & $1.0 \times 10^{0}$ \\
\hline $05-10$ & 13 & $3.4 \times 10^{-1}$ & $2.4 \times 10^{1}$ & $3.7 \times 10^{0}$ & $4.9 \times 10^{0}$ & $6.2 \times 10^{0}$ & $1.0 \times 10^{0}$ & $1.2 \times 10^{0}$ \\
\hline $10-15$ & 13 & $1.4 \times 10^{1}$ & $5.1 \times 10^{0}$ & $7.9 \times 10^{-1}$ & $1.9 \times 10^{0}$ & $1.9 \times 10^{0}$ & $5.7 \times 10^{-2}$ & $1.2 \times 10^{0}$ \\
\hline $15-25$ & 9 & $5.2 \times 10^{-2}$ & $2.1 \times 10^{0}$ & $9.8 \times 10^{-1}$ & $1.0 \times 10^{0}$ & $7.0 \times 10^{-1}$ & $-3.9 \times 10^{-1}$ & $1.2 \times 10^{0}$ \\
\hline $25-40$ & 4 & $2.8 \times 10^{-2}$ & $9.1 \times 10^{-1}$ & $4.9 \times 10^{-1}$ & $4.8 \times 10^{-1}$ & $4.2 \times 10^{-1}$ & $-1.4 \times 10^{0}$ & $1.6 \times 10^{0}$ \\
\hline $40-60$ & 2 & $4.7 \times 10^{-2}$ & $2.0 \times 10^{-1}$ & $1.2 \times 10^{-1}$ & $1.2 \times 10^{-1}$ & $1.1 \times 10^{-1}$ & $-2.3 \times 10^{0}$ & $1.0 \times 10^{0}$ \\
\hline $0-05$ & 31 & $3.0 \times 10^{-1}$ & $3.0 \times 10^{1}$ & $1.1 \times 10^{1}$ & $1.2 \times 10^{1}$ & $6.9 \times 10^{0}$ & $2.2 \times 10^{0}$ & $1.0 \times 10^{0}$ \\
\hline $0-10$ & 13 & $3.2 \times 10^{-1}$ & $2.4 \times 10^{1}$ & $7.0 \times 10^{\circ}$ & $8.2 \times 10^{0}$ & $6.1 \times 10^{0}$ & $1.8 \times 10^{0}$ & $1.0 \times 10^{0}$ \\
\hline $0-15$ & 12 & $3.3 \times 10^{-1}$ & $1.6 \times 10^{1}$ & $5.0 \times 10^{0}$ & $6.0 \times 10^{0}$ & $4.1 \times 10^{0}$ & $1.5 \times 10^{0}$ & $9.6 \times 10^{-1}$ \\
\hline $0-25$ & 8 & $5.9 \times 10^{-1}$ & $4.6 \times 10^{0}$ & $3.6 \times 10^{0}$ & $3.3 \times 10^{0}$ & $1.3 \times 10^{0}$ & $1.1 \times 10^{0}$ & $6.7 \times 10^{-1}$ \\
\hline $0-40$ & 4 & $7.1 \times 10^{-1}$ & $2.7 \times 10^{0}$ & $2.1 \times 10^{0}$ & $1.9 \times 10^{0}$ & $8.9 \times 10^{-1}$ & $5.3 \times 10^{-1}$ & $6.1 \times 10^{-1}$ \\
\hline $0-60$ & 2 & $5.4 \times 10^{-1}$ & $1.2 \times 10^{0}$ & $8.7 \times 10^{-1}$ & $8.7 \times 10^{-1}$ & $4.7 \times 10^{-1}$ & $-2.2 \times 10^{-1}$ & $5.7 \times 10^{-1}$ \\
\hline
\end{tabular}

NOTE:Specific activity is decay corrected to 1994 .

$\mathrm{N}^{\mathrm{a}}$ stands for number of individual samples. 
Table C-20.Americium-241 radionuclide concentration summary for all soil profiles taken from the 1978 NMIRS together with our most recent trip in 1989 on Mellu Island (F23).

\begin{tabular}{|c|c|c|c|c|c|c|c|c|}
\hline \multirow{2}{*}{$\begin{array}{c}\text { Soil } \\
\text { depth } \\
\text { (cm) }\end{array}$} & \multirow[b]{2}{*}{$\mathrm{Na}^{\mathrm{a}}$} & \multicolumn{5}{|c|}{ pCi g-1 dry wt } & \multirow{2}{*}{$\begin{array}{c}\text { Mean } \\
\text { of logs }\end{array}$} & \multirow{2}{*}{$\begin{array}{c}\text { SD } \\
\text { of logs } \\
\end{array}$} \\
\hline & & Minimum & Maximum & Median & Mean & SD & & \\
\hline $0-05$ & 13 & $1.5 \times 10^{0}$ & $2.5 \times 10^{1}$ & $7.5 \times 10^{0}$ & $9.2 \times 10^{0}$ & $6.6 \times 10^{0}$ & $1.9 \times 10^{0}$ & $8.2 \times 10^{-1}$ \\
\hline $05-10$ & 12 & $1.2 \times 10^{0}$ & $1.5 \times 10^{1}$ & $3.4 \times 10^{0}$ & $5.6 \times 10^{0}$ & $4.7 \times 10^{0}$ & $1.4 \times 10^{0}$ & $8.2 \times 10^{-1}$ \\
\hline $10-15$ & 10 & $9.1 \times 10^{-1}$ & $1.1 \times 10^{1}$ & $2.3 \times 10^{0}$ & $3.3 \times 10^{0}$ & $3.1 \times 10^{0}$ & $9.2 \times 10^{-1}$ & $7.5 \times 10^{-1}$ \\
\hline $15-25$ & 9 & $2.6 \times 10^{-1}$ & $5.6 \times 10^{0}$ & $1.6 \times 10^{0}$ & $1.7 \times 10^{0}$ & $1.6 \times 10^{0}$ & $1.9 \times 10^{-1}$ & $9.3 \times 10^{-1}$ \\
\hline $25-40$ & 4 & $1.7 \times 10^{-1}$ & $8.8 \times 10^{-1}$ & $4.2 \times 10^{-1}$ & $4.7 \times 10^{-1}$ & $3.1 \times 10^{-1}$ & $-9.4 \times 10^{-1}$ & $7.2 \times 10^{-1}$ \\
\hline $40-60$ & 1 & $2.4 \times 10^{-2}$ & $2.4 \times 10^{-2}$ & $2.4 \times 10^{-2}$ & $2.4 \times 10^{-2}$ & $0.0 \times 10^{0}$ & $-3.7 \times 10^{0}$ & $0.0 \times 10^{0}$ \\
\hline $0-05$ & 13 & $1.5 \times 10^{0}$ & $2.5 \times 10^{1}$ & $7.5 \times 10^{0}$ & $9.2 \times 10^{0}$ & $6.6 \times 10^{0}$ & $1.9 \times 10^{0}$ & $8.2 \times 10^{-1}$ \\
\hline $0-10$ & 12 & $1.4 \times 10^{0}$ & $1.9 \times 10^{1}$ & $7.6 \times 10^{0}$ & $7.6 \times 10^{0}$ & $4.7 \times 10^{0}$ & $1.8 \times 10^{0}$ & $6.9 \times 10^{-1}$ \\
\hline $0-15$ & 9 & $1.3 \times 10^{0}$ & $1.5 \times 10^{1}$ & $6.8 \times 10^{0}$ & $6.5 \times 10^{0}$ & $4.1 \times 10^{0}$ & $1.7 \times 10^{0}$ & $7.4 \times 10^{-1}$ \\
\hline $0-25$ & 7 & $2.1 \times 10^{0}$ & $9.5 \times 10^{0}$ & $4.5 \times 10^{0}$ & $4.8 \times 10^{0}$ & $2.8 \times 10^{0}$ & $1.4 \times 10^{0}$ & $5.9 \times 10^{-1}$ \\
\hline $0-40$ & 3 & $1.4 \times 10^{0}$ & $3.3 \times 10^{0}$ & $1.7 \times 10^{0}$ & $2.1 \times 10^{0}$ & $1.0 \times 10^{0}$ & $6.8 \times 10^{-1}$ & $4.5 \times 10^{-1}$ \\
\hline $0-60$ & 1 & $9.4 \times 10^{-1}$ & $9.4 \times 10^{-1}$ & $9.4 \times 10^{-1}$ & $9.4 \times 10^{-1}$ & $0.0 \times 10^{0}$ & $-6.6 \times 10^{-2}$ & $0.0 \times 10^{0}$ \\
\hline
\end{tabular}

NOTE: Specific activity is decay corrected to 1994.

$\mathrm{N}^{\mathrm{a}}$ stands for number of individual samples. 
Appendix D

Maps of the Islands 


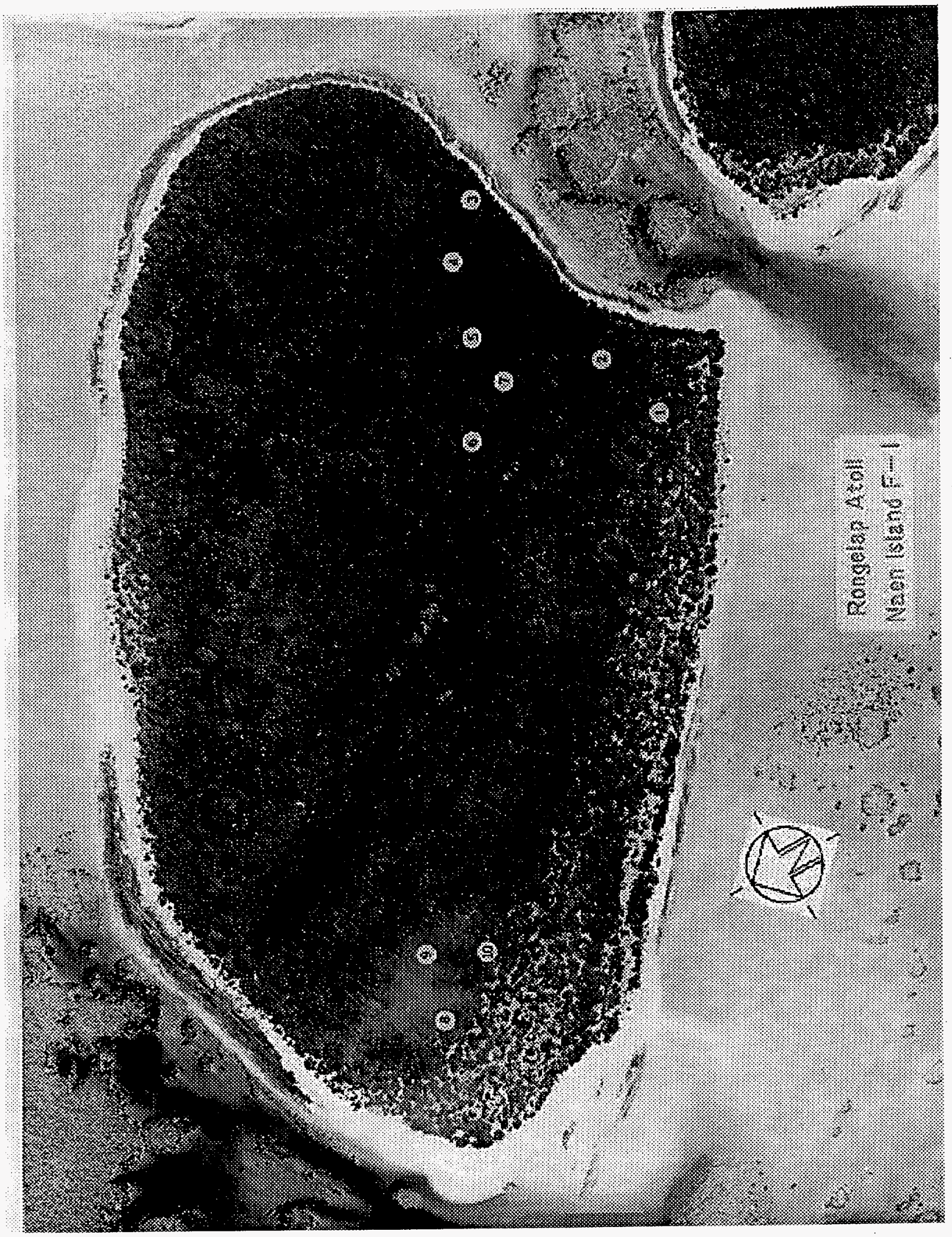




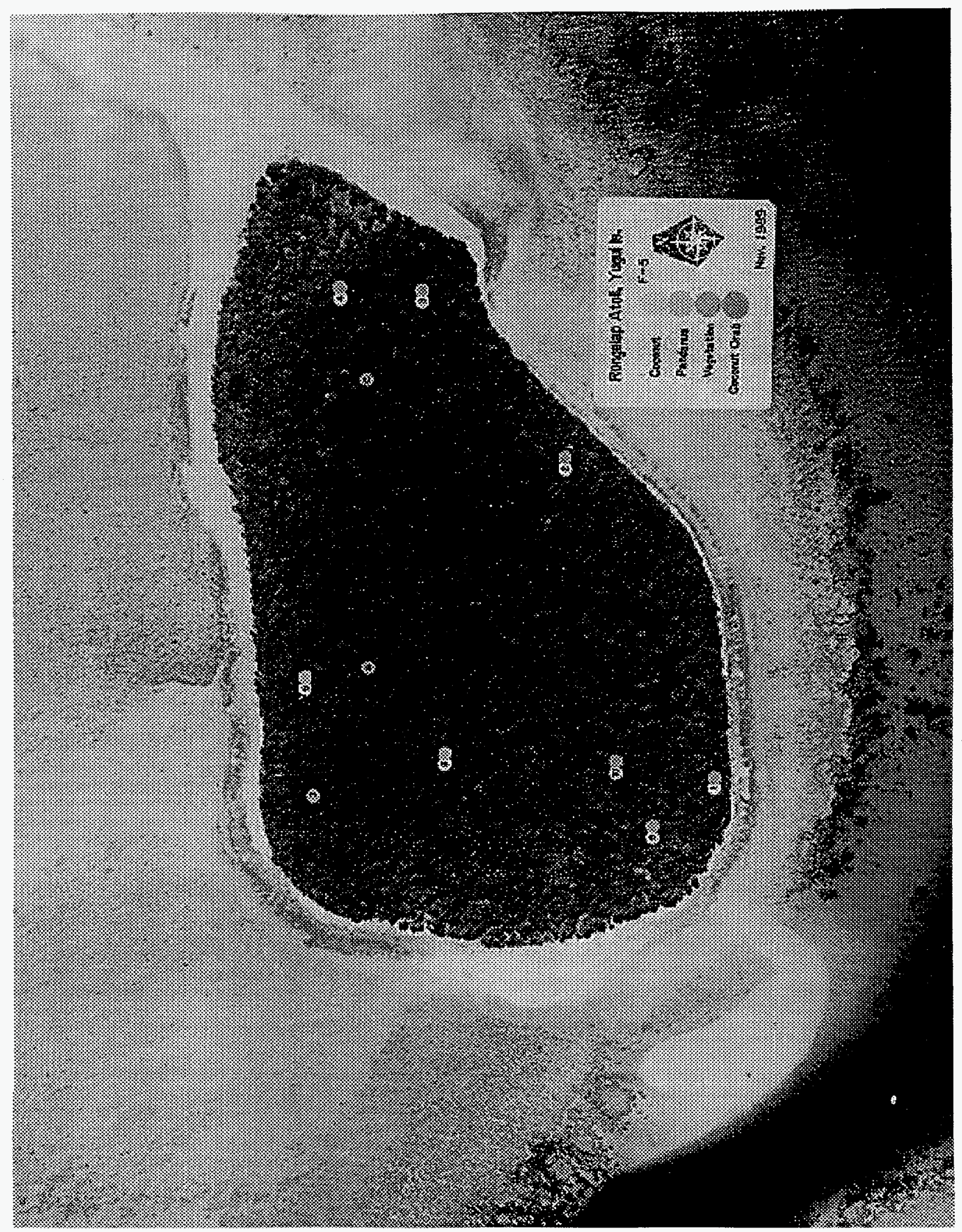




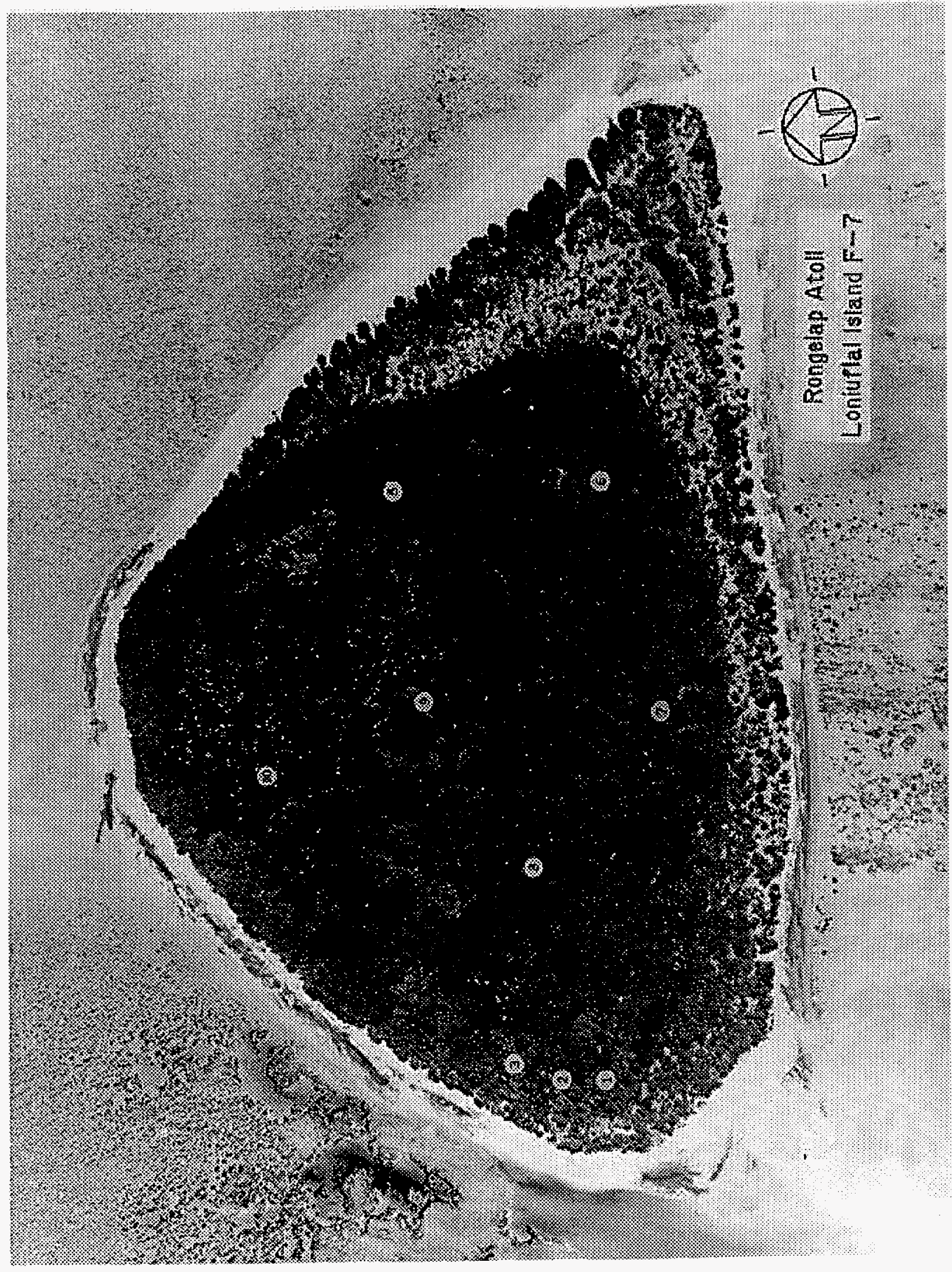




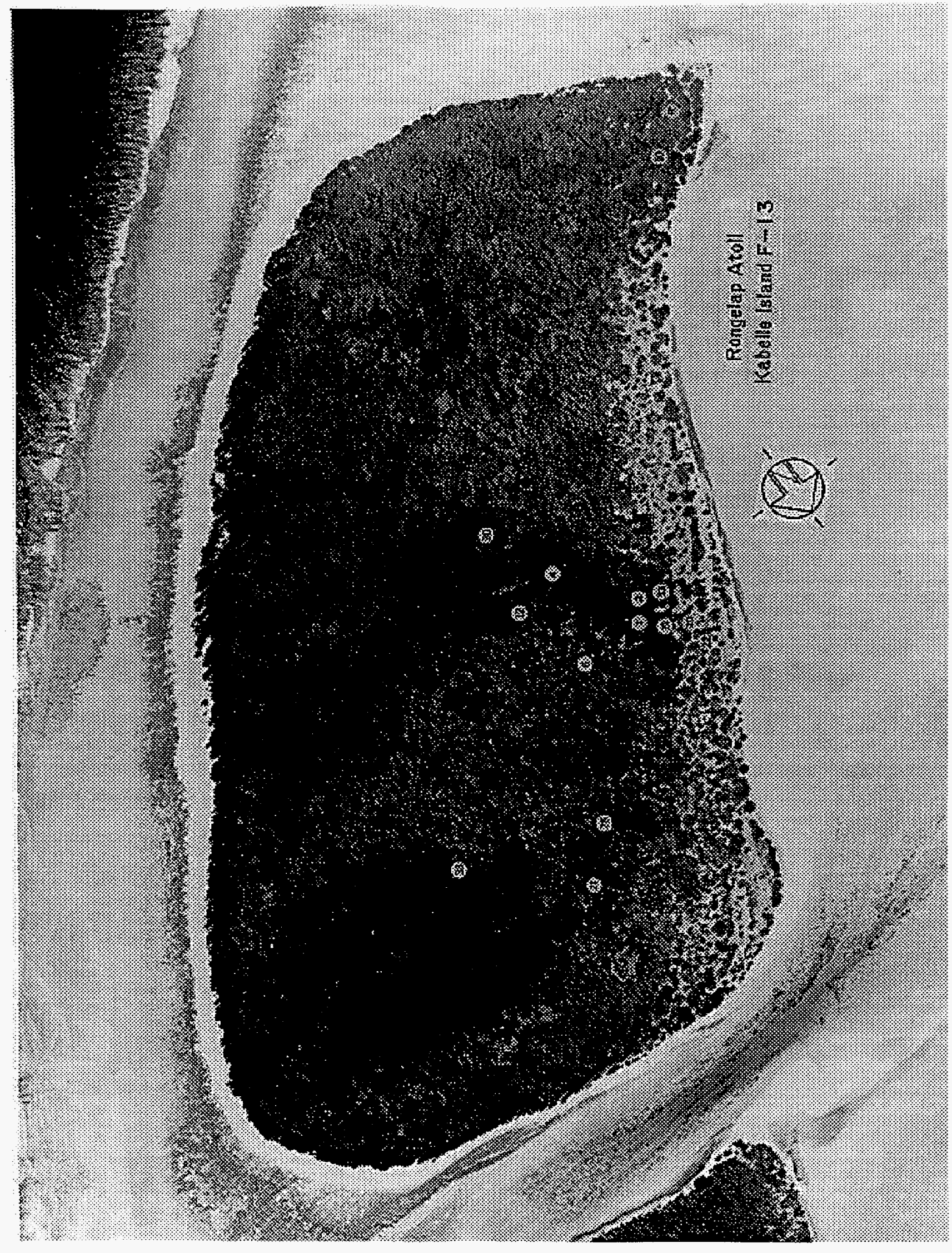




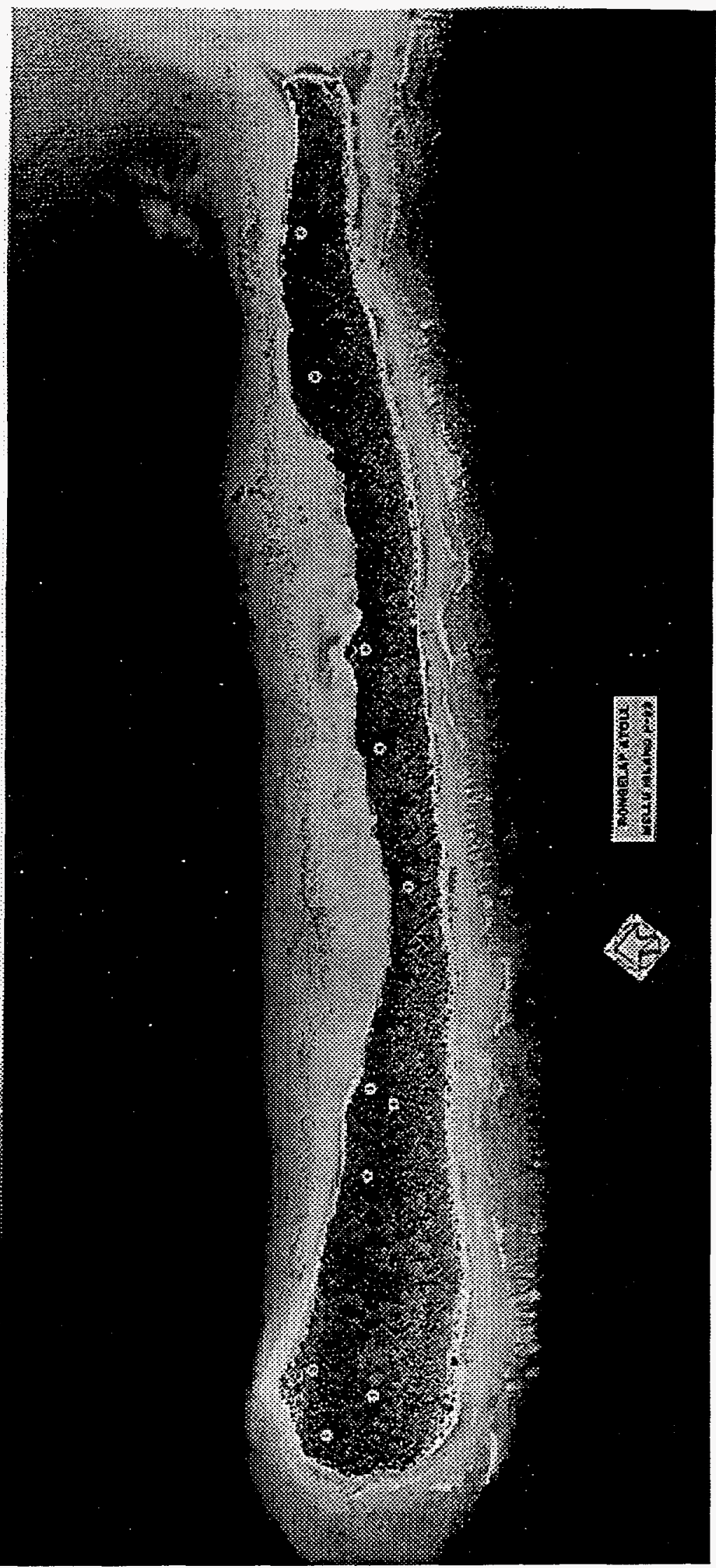


